\title{
EVOLUTIONARY STRATEGY DYNAMICS FOR TAG-BASED COOPERATION AND DEFECTION IN THE SPATIAL AND ASPATIAL SNOWDRIFT GAME
}

\author{
ROBERT A. LAIRD \\ Department of Biological Sciences, \\ University of Lethbridge, Canada \\ robert.laird@uleth.ca
}

Received June 29, 2011; Revised October 19, 2011

\begin{abstract}
Cooperation is a costly behavior undertaken by one individual which benefits another individual. Since cooperators are easily exploited by defectors (those who receive the benefits of cooperation but do not cooperate themselves), the evolution and maintenance of cooperation rely on mechanisms that allow cooperators to interact with one another more frequently than would be predicted based on their relative abundance in a population. One simple mechanism is based on the recognition of "tags" - arbitrary, yet identifiable phenotypic traits. Tags allow for the existence of conditionally cooperative strategies; e.g. individuals could adopt a strategy whereby they cooperate with tag-mates but defect against non-tag-mates. Previous research has considered the tag and strategy dynamics of unconditional and conditional strategies engaged in the Prisoner's Dilemma game, the paradigmatic framework for studying the evolutionary dynamics of cooperation, in which defection against a cooperator yields the greatest fitness payoff, followed by mutual cooperation, mutual defection, and cooperation with a defector. Here, using complementary spatial and aspatial lattice models, an alternative payoff structure is considered, based on the Snowdrift game, in which the rankings of the payoffs associated with mutual defection and cooperation with a defector are reversed relative to the Prisoner's Dilemma. In the absence of mutation, it is demonstrated that the aspatial two-tag game tends to collapse into the traditional, non-tag-based Snowdrift game, with the frequency of cooperators and defectors predicted precisely by evolutionary dynamics analysis. The spatial two-tag game, on the other hand, produces a richer variety of outcomes, whose occurrence depends on the cost-benefit ratio of mutual cooperation; these outcomes include the dominance of conditional cooperators, the dominance of unconditional defectors, and the cyclic (or noncyclic) coexistence of the two. These outcomes are then shown to be modified by mutation (which softens the transition boundaries between outcomes), and by the presence of more than two tags (which promotes nepotistic conditional cooperation).
\end{abstract}

Keywords: Evolutionary game theory; green-beard effect; Prisoner's Dilemma; Snowdrift game; spatial model.

\section{Introduction}

Cooperation occurs when an individual's actions benefit another at a cost to itself [Nowak, 2006b]. The evolution of cooperation remains a central theme in evolutionary biology because of its importance in the history of life: evolutionary transitions from one level of biological organization to the next typically require cooperation [Maynard Smith \& Szathmáry, 1997, 1999]. More generally, efforts to understand the evolution of 
cooperation have greatly contributed to the recent boom in evolutionary game theory, an interdisciplinary approach that spans such disparate fields as biology, statistical physics, economics and sociology (reviewed in [Hofbauer \& Sigmund, 1998; Nowak, 2006a; Szabó \& Fáth, 2007; Roca et al., 2009b; Perc \& Szolnoki, 2010; Gintis, 2009]). Despite this importance, from the outset, cooperative systems seem inherently fragile. Specifically, they are susceptible to exploitation by defectors - cheaters who receive benefits from cooperators but fail to return the favor [Nowak, 2006b]. Efforts to explain cooperation, therefore, have focused on mechanisms that allow such exploitation to be avoided or mitigated. The green-beard effect [Hamilton, 1964; Dawkins, 1976] is the whimsical name given to one of the simplest proposed mechanisms of cooperation (for recent reviews of other mechanisms, see [Nowak, 2006b; Sherratt \& Wilkinson, 2009; Nowak \& Highfield, 2011]). In Dawkins' [1976] formulation, a gene simultaneously coding for (a) a conspicuous and recognizable phenotypic "tag" (e.g. a green beard), (b) the propensity to cooperate with others who also bear the tag, can spark a cooperative revolution within a population. Dawkins himself raised two potential problems with such a system. First, it seemed unrealistic for a single gene to be able to code for such wildly different traits [Dawkins, 1976]. Second, if the traits were instead coded by different genes (a scenario sometimes called the "armpit effect"), then green-bearded cooperators could be exploited by defectors masquerading as cooperators - individuals who sport a green beard but do not cooperate [Dawkins, 1982]. Notwithstanding these initial misgivings, empirical examples of green-beards are starting to accumulate [Keller \& Ross, 1998; Queller et al., 2003; Smukalla et al., 2008], leading to renewed interest in the theory underpinning tag-based cooperation. Riolo et al. [2001, 2002] showed that tag-based cooperation can evolve when individuals with identical tags always cooperate, but Roberts and Sherratt [2002] demonstrated that tag-based cooperation is undermined when even identically tagged individuals can defect against one another. Further work, however, has shown that tag-based cooperation can emerge - even in the presence of "unconditional defectors" - under a variety of ecological and behavioral scenarios [Traulsen \& Schuster, 2003; Axelrod et al., 2004; Hammond \& Axelrod, 2006b, 2006a; Jansen \& van Baalen, 2006;
Masuda \& Ohtsuki, 2007; Traulsen \& Nowak, 2007; Traulsen, 2008; Antal et al., 2009; Lima et al., 2009; Laird, 2011; reviewed in Szabó \& Fáth, 2007]. These previous papers considered a frequency-dependent strategy fitness structure defined by the stalwart of cooperation theory, the Prisoner's Dilemma game. However, other fitness structures are possible, potentially enriching the diversity of evolutionary outcomes of tag-based cooperation. To this end, complementary spatial and aspatial simulation models are used to explore the evolutionary strategy dynamics of tag-based cooperation and defection in the Snowdrift game [Maynard Smith, 1982; Sugden, 1986; Doebeli \& Hauert, 2005], focusing on the two-tag version of the models, in the absence of mutation. I report that the aspatial two-tag game tends to collapse to the traditional, non-tagbased Snowdrift game, with the frequency of cooperators and defectors predicted precisely by evolutionary dynamics analysis. The spatial two-tag game, on the other hand, allows a richer diversity of outcomes, which are dependent on the costbenefit ratio of mutual cooperation; these outcomes include the dominance of green-beard cooperators, the dominance of unconditional defectors, and the cyclic (or noncyclic) coexistence of the two. I then show how these outcomes are modified by mutation (which softens the transition boundaries between outcomes), and by the presence of more than two tags (which promotes nepotistic conditional cooperation).

\section{Snowdrift Game}

Along with the Prisoner's Dilemma [Axelrod \& Hamilton, 1981; Axelrod, 2006], the Snowdrift game (a.k.a. "Chicken", "Hawk-Dove"; [Maynard Smith, 1982; Sugden, 1986]) is one of the most prominent frameworks used by evolutionary game theorists to study the evolution of cooperation (reviewed in [Nowak \& Sigmund, 2004; Doebeli \& Hauert, 2005]), and recently a growing number of studies have pushed the Snowdrift game towards new research frontiers (e.g. [Hauert \& Doebeli, 2004; Wang et al., 2006; Perc, 2007; Du et al., 2009; Szolnoki \& Perc, 2008, 2009; Roca et al., 2009a; Chen \& Wang, 2010]). As is typical with fixed-strategy, twoplayer games, players of the Snowdrift game either cooperate with $(C)$ or defect against $(D)$ each other. In the Snowdrift game, cooperation is costly yet both players benefit relative to mutual defection 
(the worst possible outcome) so long as at least one player cooperates. The game's name alludes to the story of two individuals whose car is stuck in a Snowdrift. Each individual can either help shovel the car out of the drift (cooperate) or remain in the car (defect). It is best to remain in the car while one's co-player shovels, thus acquiring the benefits of cooperation (freedom from the drift) without paying the costs (shoveling). Second best is mutual cooperation; both players receive the benefit, but the cost is shared. Third best is to shovel while one's co-player remains in the car; the focal individual has to do all the work, but at least he or she is free of the drift. Finally, the worst outcome is mutual defection, because both individuals remain stuck and cannot get home. (As an aside, in most depictions, the two players are described as driving in two separate cars, but the analogy actually works better if the players are in the same car; otherwise, one player could presumably dig out his or her car while leaving the other car bedrifted.) In biological systems, this might arise, for example, in vigilance behavior in prey: if at least one of the two players is vigilant for predators (= cooperates) both players benefit; however, the costs of cooperation (e.g. lost foraging opportunities, increased exposure to predators) are borne only by those who cooperate. Another example comes from microbial ecology, where the production of compounds to aid in extracellular digestion is a cooperative action that also provides a benefit to the producer [Sherratt et al., 2009]. These examples link the Snowdrift game to a broader class of $n$-player Public Goods games (e.g. see discussion in [Souza et al., 2009; Sherratt \& Wilkinson, 2009]).

In mathematical terms, in the Snowdrift game, cooperation yields a benefit of $b$ to both players at a total cost of $c(b>c>0)$, while defection is cost-free but yields no benefit. The game's net payoffs, which are equated with evolutionary fitness [Maynard Smith, 1982], depend on the combination of strategies adopted by the players, setting the stage for frequency-dependent selection and nonlinear dynamics. In the case of mutual cooperation both players receive the benefit and share the cost of cooperation, and therefore receive a net payoff of $a_{C C}=b-c / 2$. Mutual defection results in a net payoff of $a_{D D}=0$. When one player cooperates and another defects, the defector gets the benefit without paying a cost for a net payoff of $a_{D C}=b$; the cooperator still receives the benefit, but is stuck with the whole cost for a net payoff of $a_{C D}=b-c$. Therefore, the payoffs in the Snowdrift game are ranked $a_{D C}>a_{C C}>a_{C D}>a_{D D}$, as in the description above. Critically, this implies that cooperators can invade a population of defectors and vice versa [Hofbauer \& Sigmund, 1998]. In the absence of tags, this means that in contrast to the Prisoner's Dilemma (in which the inequality between $a_{C D}$ and $a_{D D}$ is reversed), in large, well-mixed populations playing the Snowdrift game, cooperators and defectors coexist stably, with an equilibrium frequency of cooperators of $x_{C}^{*}=\left(a_{D D}-a_{C D}\right) /\left(a_{C C}-a_{C D}-\right.$ $\left.a_{D C}+a_{D D}\right)$ and an equilibrium frequency of defectors of $x_{D}^{*}=1-x_{C}^{*}$ [Hofbauer \& Sigmund, 1998; Hauert \& Doebeli, 2004; Nowak, 2006a].

\section{Tags and Strategies}

The existence of arbitrarily different types of individuals allows for additional fixed strategies, beyond the traditional $C$ and $D$. Consider individuals that have one of $m$ arbitrary tags. (For most of the analyses described here, $m=2$, the simplest tag-based system, although $m=5$ is also considered briefly as an example of a multitag system.) Each tag is outwardly and easily identifiable by everyone. Independent of tag, in the present study individuals employ one of four strategies $(C, I, E$, and $D$; see [Traulsen, 2008; Lima et al., 2009; Laird, 2011]) that determine whether they will cooperate with or defect against each of the various tags (other fixed strategies are possible for $m>2$, but these are not considered here). Unlike tags, individuals' strategies are hidden and are only apparent in retrospect, i.e. after an interaction has taken place. In the current formulation, individuals have no memory or ability to "image score", precluding direct reciprocity [Axelrod \& Hamilton, 1981; Axelrod, 2006], indirect reciprocity [Nowak \& Sigmund, 1998], or other memory-based conditional strategies [Wang et al., 2006]. Both tag and strategy are deterministic and are fixed for an individual's whole life. Individuals using strategy $C$ are "unconditional cooperators", who cooperate with everyone irrespective of tag. Individuals using strategy $D$ are "unconditional defectors", who defect against everyone irrespective of tag. Individuals using strategy $I$ or $E$ are conditional cooperators of a nepotistic or traitorous flavor, respectively. Those using strategy I are "intra-tag cooperators", who cooperate with tag-mates but defect against 
non-tag-mates. Those using strategy $E$ are "extra-tag cooperators", who cooperate with non-tag-mates but defect against tag-mates.

Thus, the two-tag payoff matrix, $\mathbf{A}=\left[A_{i j}\right]$ is given by the following:

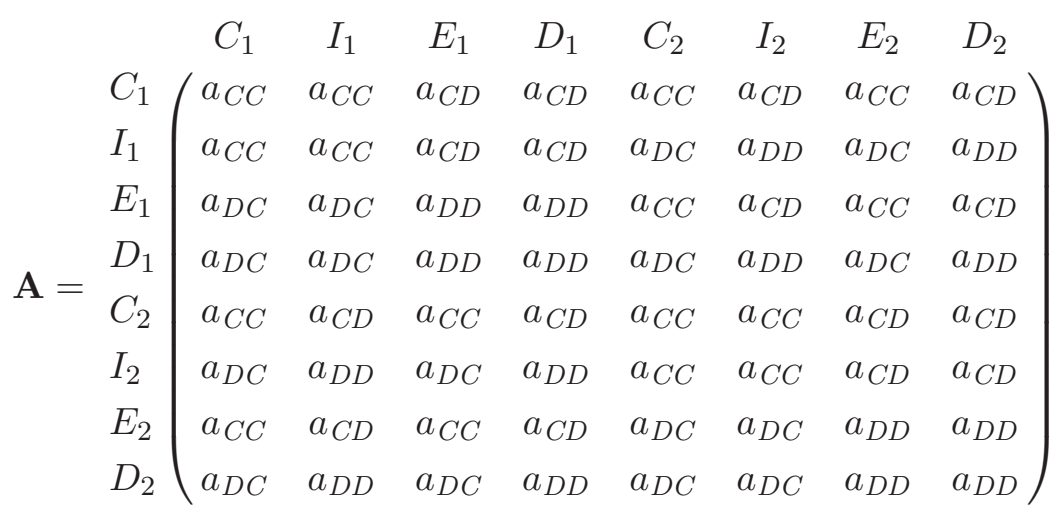

where $A_{i j}$ gives the payoff of an individual of the tag (subscript number) and strategy (letter) of row $i$ against an individual of the tag and strategy of column $j$. The A matrix can be simplified by substituting $a_{C C}=1, a_{D D}=0, a_{C D}=1-r$, and $a_{D C}=1+r$, where $r=c /(2 b-c)$ is the costbenefit ratio of mutual cooperation $(0<r<1$; [Hauert \& Doebeli, 2004; Doebeli \& Hauert, 2005]). Thus, in the limit of large, well-mixed populations, and in the absence of tags, $x_{C}^{*}=1-r$ and $x_{D}^{*}=r$ [Hauert \& Doebeli, 2004].

Note that other recent studies have typically considered a subset of the strategies listed here. Specifically, most have used either $I$ and $D$ (termed the "cooperative tag mechanism" by Traulsen [2008]; e.g. [Jansen \& van Baalen, 2006; Traulsen \& Nowak, 2007]) or $I$ and $C$ (termed the "defective tag mechanism" by Traulsen [2008]; e.g. [Riolo et al., 2001; Traulsen \& Schuster, 2003]). Traulsen [2008] considered the combination of three strategies, $C, I$ and $D$. However, including all four strategies is appropriate because $E$ can presumably arise through mutation from $C$ or $D$ just as easily as $I$, and because $E$-strategists, despite their "traitorousness", can evolve to dominance under certain scenarios [Laird, 2011]. A small number of recent studies have included all four strategies [Axelrod et al., 2004; Hammond \& Axelrod, 2006b, 2006a; Lima et al., 2009; Laird, 2011], but these considered the Prisoner's Dilemma game rather than the Snowdrift game.

\section{Spatial and Aspatial Simulations}

Local network effects can have strong effects on the coexistence and exclusion conditions of cooperative and defective strategies [Nowak \& May, 1992, 1993;
Nowak et al., 1994b, 1994a; Hauert, 2002, 2006; Lieberman et al., 2005; Szabó \& Fáth, 2007; Taylor et al., 2007; Langer et al., 2008; Roca et al., 2009b, 2009a], including in tag-based systems [Jansen \& van Baalen, 2006; Laird, 2011]. Spatial networks, in which interactions occur in spatially local neighborhoods, have received particular attention. For example, space can promote the coexistence of cooperators and defectors in the non-tag-based Prisoner's Dilemma game [Nowak \& May, 1992, 1993; Nowak et al., 1994b, 1994a]. In the spatial extension of the non-tag-based Snowdrift game, however, cooperators typically persist at lower relative abundances, and over a smaller range of $r$, than predicted by evolutionary dynamics [Hauert \& Doebeli, 2004; but see Roca et al., 2009a]. Analytically speaking, it is difficult to predict how spatially explicit interactions will affect strategy (and tag) coexistence [Szabó \& Fáth, 2007]. Hence, simulation is a valuable tool for investigating the role of space.

The spatial model described here (also see [Laird, 2011]) builds upon previous (nontag) models of the evolution of cooperation, in particular [Hauert \& Doebeli, 2004]. Space is represented by a square $L \times L$ lattice with periodic boundaries (i.e. a torus). A single individual belonging to one of the eight tag-by-strategy combinations (i.e. for $m=2$ ) occupies each cell. Initially, every cell has an equal probability of being occupied by each category. Cells are selected randomly for potential replacement, with $L^{2}$ such events defined as one generation (i.e. on average, every cell is selected for potential replacement once per generation). Typically, the models are run on $L^{2}=10000$ cell lattices for 20000 generations for a total of 
$2 \times 10^{8}$ interactions per value of $r$ (larger lattices and longer generation times are also examined for a subset of the analyses in order to pin down the location and nature of observed phase transitions; see Sec. 5). Each time a focal cell is selected, a competitor cell is also randomly selected from the four cells in the focal cell's von Neumann neighborhood (i.e. the cells immediately North, South, East, and West of the focal cell [Durrett \& Levin, 1994]). The average payoffs to focal and competitor cells from their respective von Neumann neighborhoods are then compared. These are called $p_{x}$ and $p_{y}$, respectively. If $p_{y}>p_{x}$, an asexual clone of the occupant of the competitor cell replaces the occupant of the focal cell with a probability $\left(p_{y}-p_{x}\right) /(1+r)$; the denominator scales this probability between zero and one. If $p_{y} \leq p_{x}$, the focal cell is unchanged. (This update rule is known as the "replicator rule" due to its convergence with replicator dynamics in the limit of large, well-mixed populations, see [Hauert \& Doebeli, 2004; Roca et al., 2009a]; other rules are summarized in [Szabó \& Fáth, 2007; Roca et al., 2009b]). Lastly, mutation occurs with probability $\mu$. If a mutation occurs, the occupant of the focal cell changes its tag and strategy to a randomly chosen tag and strategy. Here I focus on the situation where there is no mutation $(\mu=0)$, but I also briefly consider nonzero mutation, as mutation has been found to alter the outcomes of spatial games (e.g. [Helbing et al., 2010a]).

In addition to the spatial version of the model, I ran a complementary aspatial version of the model. In the aspatial version, the only difference is that all the cells are selected randomly from the entire lattice, rather than from the local neighborhood.

\section{Results}

\subsection{Interpreting average tag and strategy frequencies: Two-tag model}

Figure 1 shows the main results for the spatial and aspatial simulation models for three mutation rates

(a)

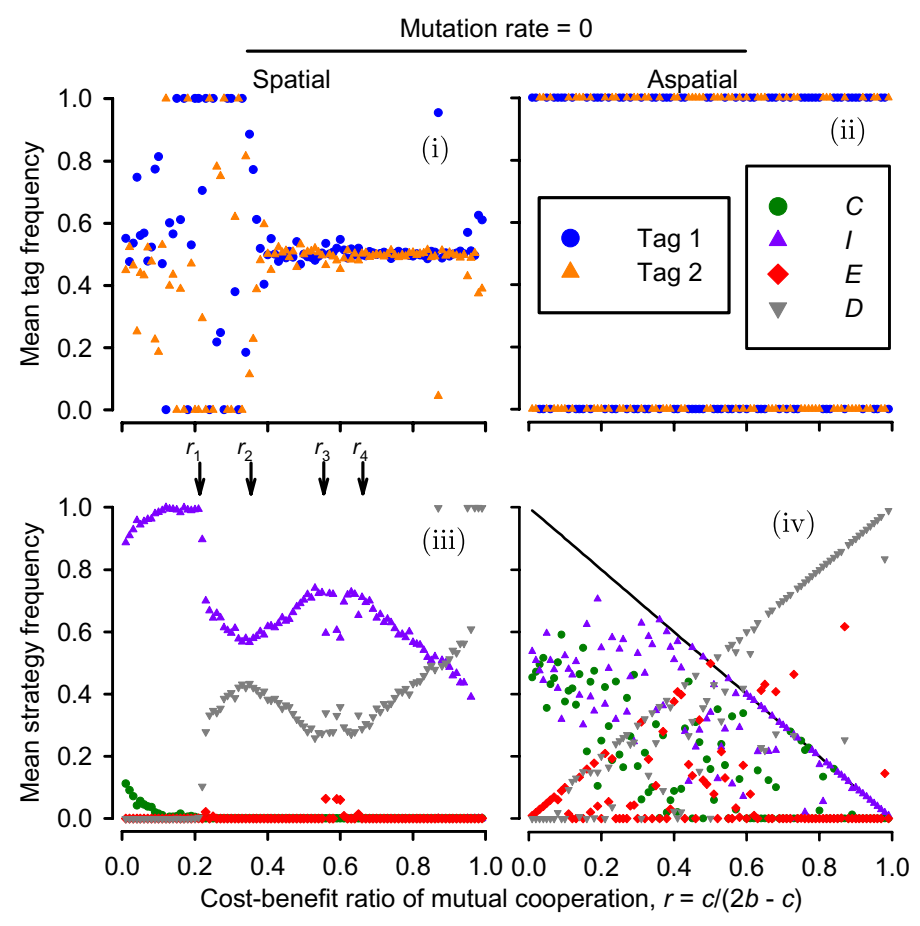

Fig. 1. Average tag [parts (i) and (ii)] and strategy relative abundances [parts (iii) and (iv)] for 5000 generations, starting after generation 15000, for the spatial [left column; parts (i) and (iii)] and aspatial [right column; parts (ii) and (iv)] twotag Snowdrift game. Both the spatial and aspatial versions of the model had a fixed population size of 10000 . Initially, every individual was given a random tag and strategy. Colors: Tag $1=$ blue circles, Tag $2=$ orange upward-triangles, unconditional cooperators $(C)=$ green circles, intra-tag cooperators $(I)=$ purple upward-triangles, extra-tag cooperators $(E)=$ red diamonds, unconditional defectors $(D)=$ gray downward-triangles. Mutation rates: (a) $\mu=0$ (no mutation), (b) $\mu=10^{-4}$, (c) $\mu=10^{-2}$. In (a)(iii), four critical values of $r\left(r_{1}, r_{2}, r_{3}\right.$ and $\left.r_{4}\right)$ are indicated. In (a)(iv), (b)(iv) and (c)(iv), the line indicates the total relative abundance of $C$ - and $I$-strategists. 
(b)
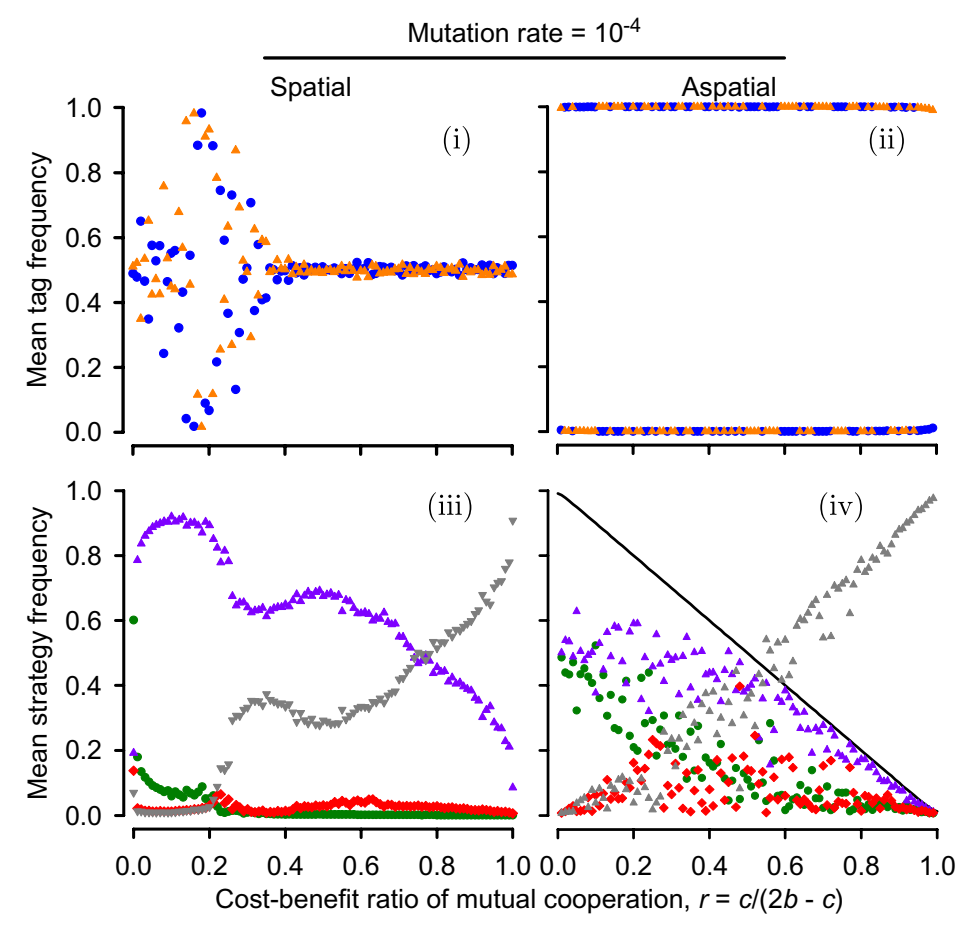

(c)
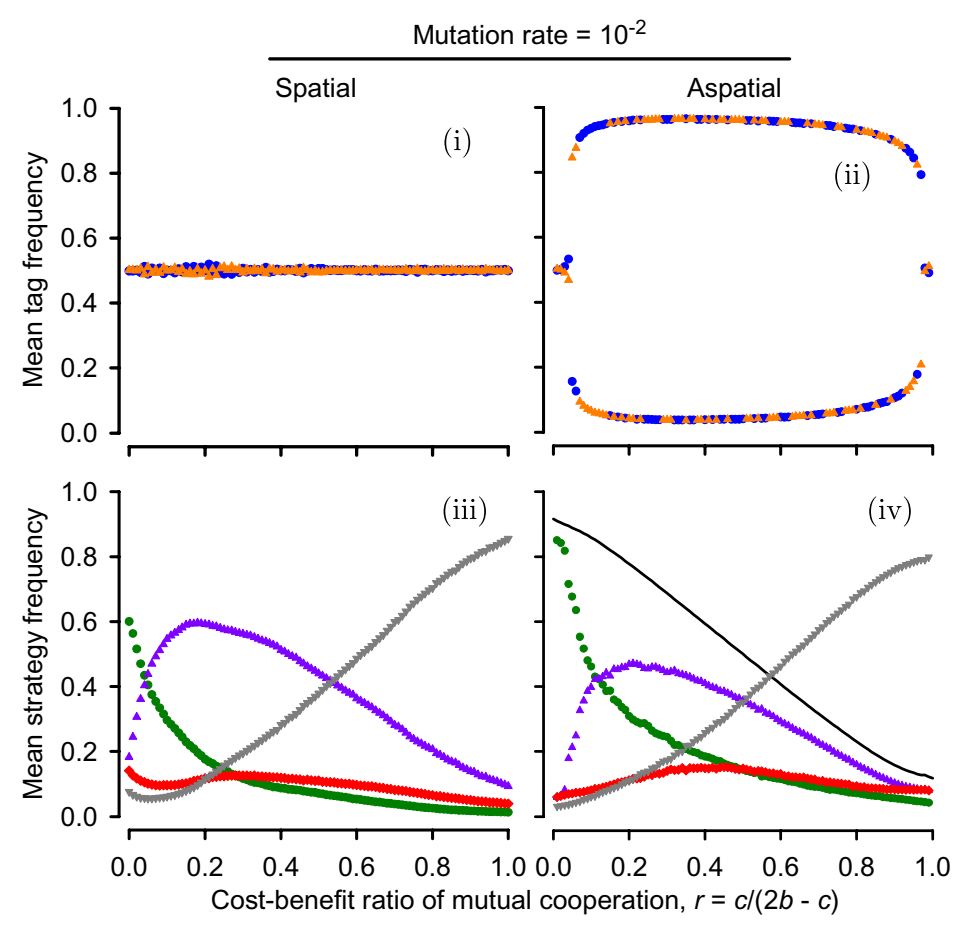

Fig. 1. (Continued)

[Fig. 1(a): $\mu=0$; Fig. 1(b): $\mu=10^{-4}$; Fig. 1(c): $\left.\mu=10^{-2}\right]$, and for 99 values of $r$, spanning the range of $r$ 's possible values (i.e. $0.01 \leq r \leq 0.99$ in increments of 0.01). Each subsection of Fig. 1 (i.e. Fig. 1(x), where "x" is "a", "b", or "c") is composed of four panels: Figs. 1(x)(i) and 1(x)(ii) show the mean frequency of Tags 1 and 2, between model generations 15000 and 20000, in the spatial and aspatial models, respectively; Figs. 1(x)(iii) and 1(x)(iv) show the mean frequency of $C-, I-, E$ - and $D$ strategists over the same generation interval, again in the spatial and aspatial models, respectively. In 


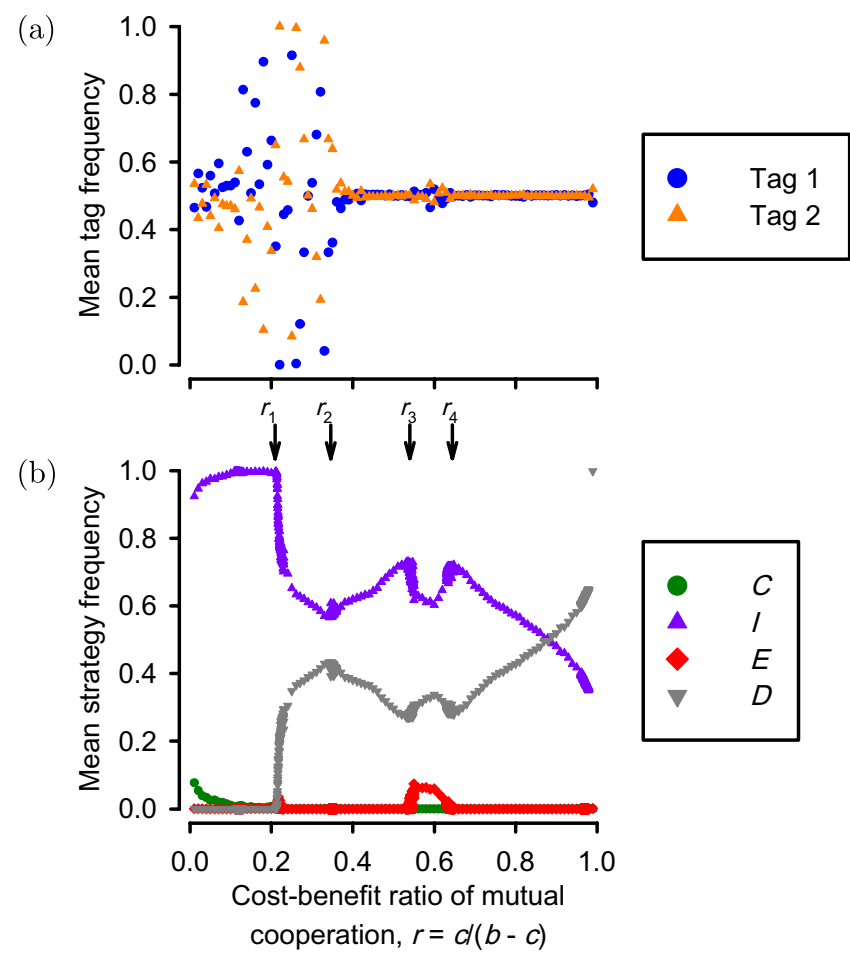

Fig. 2. (a) Average tag and (b) strategy relative abundances for 5000 generations, starting after generation 35000 , for the spatial two-tag Snowdrift game with a fixed population size of 160000 . Initially, every individual was given a random tag and strategy. Colors: Tag $1=$ blue circles, Tag $2=$ orange upward-triangles, unconditional cooperators $(C)=$ green circles, intra-tag cooperators $(I)=$ purple upward-triangles, extra-tag cooperators $(E)=$ red diamonds, unconditional defectors $(D)=$ gray downward-triangles. Mutation rate: $\mu=0$ (no mutation).

Fig. 1 , the lattice size is $100 \times 100\left(L^{2}=10000\right.$ individuals).

Figure 2 repeats the results from Figs. 1(a)(i) and 1(a)(iii) (i.e. tag and strategy frequencies for the two-tag spatial model with $\mu=0$ ), except the lattices are much larger $\left(400 \times 400 ; L^{2}=160000\right)$ and the tag and strategy frequencies are averaged after a much longer relaxation period (i.e. between generations 45000 and 50000 ). The same 99 values of $r$ as in Fig. 1 are examined in Fig. 2; however, in Fig. 2, an additional 101 values of $r$ are examined near each of six $r$-values of special interest $(0.11 \leq r \leq 0.13,0.21 \leq r \leq 0.23,0.34 \leq r \leq 0.36$, $0.53 \leq r \leq 0.55,0.63 \leq r \leq 0.65$ and $0.96 \leq r \leq$ 0.98 , all in increments of 0.0002 ), for a total of 705 $r$-values. These extra analyses enable a more precise characterization of the qualitatively different outcomes that are observed at different $r$-values in the spatial two-tag Snowdrift game (see Sec. 5.3).
In the sections that follow, I provide detailed results and examples for the dynamics of the spatial and aspatial two-tag Snowdrift game in the absence of mutation, and then briefly touch upon the cases of relatively low and high mutation rates, as well as the five-tag case.

\subsection{Simulation results in the absence of mutation: Aspatial two-tag model}

In the aspatial two-tag model with no mutation, one of the tags is inevitably excluded by the other [Fig. 1(a)(ii)]. In this situation, the remaining tag serves no purpose (i.e. effectively $C$ and $I$ are rendered equivalent, and $E$ and $D$ are rendered equivalent). Therefore, the tag-based Snowdrift game decomposes into the standard (non-tagbased) Snowdrift game. Because of the large (albeit finite) population size, and the well-mixed conditions, the outcome of the model is very closely approximated by the solution given by evolutionary dynamics analysis. Specifically, the combined frequency of $C$ - and $I$-strategists [given by the black line in Fig. 1(a)(iv)] is essentially identical to the predicted value of $1-r$ (Pearson correlation coefficient $>0.999999)$. Thus, the aspatial model reconfirms that cooperative and defective strategists can coexist in large, well-mixed populations.

\subsection{Simulation results in the absence of mutation: Spatial two-tag model}

When space is added to this scenario, a very different suite of outcomes is observed [Figs. 1(a)(i), 1(a)(iii), 2(a) and 2(b)]. Phase transitions occur at four critical values of $r\left[r_{1} \approx 0.22, r_{2} \approx 0.35\right.$, $r_{3} \approx 0.53$ and $r_{4} \approx 0.64$; Figs. 1(a)(iii) and 2(b)], which divide the parameter space into five regions with qualitatively distinct dynamics.

When $0<r<r_{1}$ (Region I), two different outcomes are possible over 20000 model generations in $100 \times 100$ lattices. In the first outcome, one of the tags excludes the other from the population, and in the alternative outcome, the tags coexist [Fig. 1(a)(i)]. In both outcomes, I-strategists dominate the population [Fig. 1(a)(iii)], although some $C$-strategists persist. ( $C$ 's persistence occurs primarily because there is no difference between $C$ and $I$ in single-tag locales.) Figures 3 and 4 show example time-series and lattice snapshots for the 


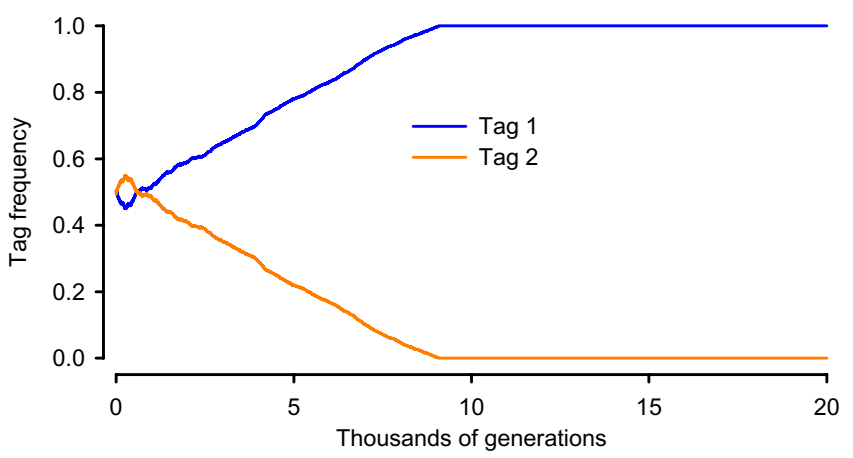

(a)

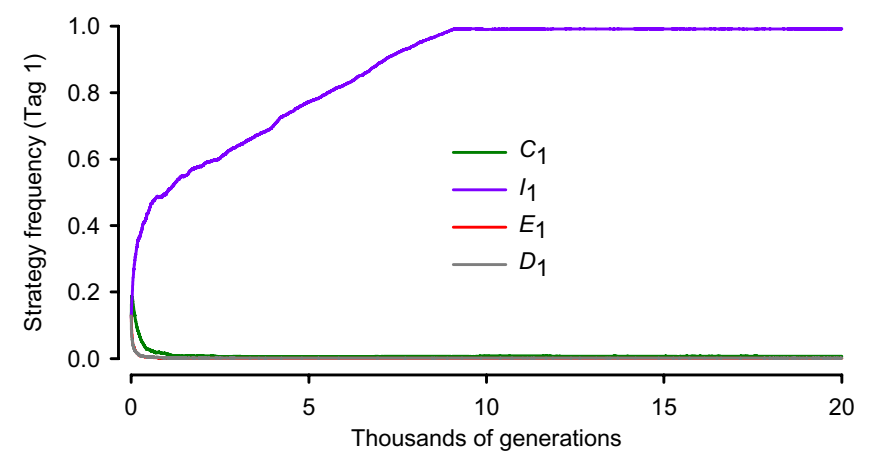

(b)

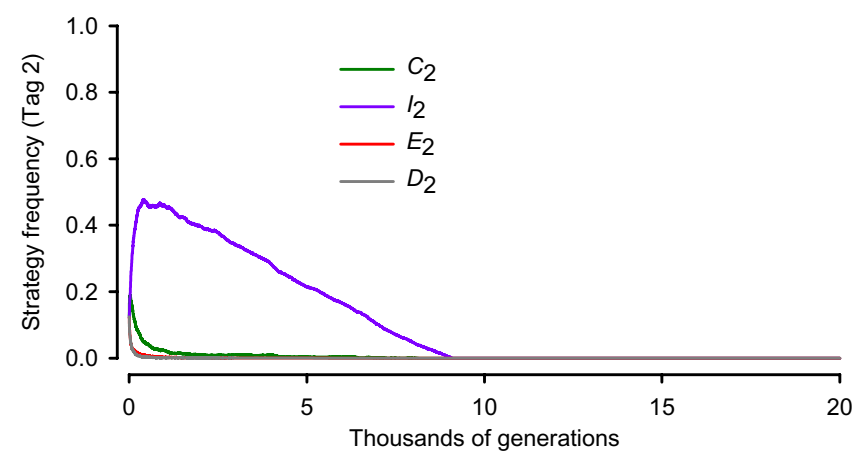

(c)

Fig. 3. Example dynamics for the spatial, two-tag Snowdrift game in the parameter region of $0<r<r_{1}$ (Region I; $r=$ 0.15 and $\mu=0$ ). (a) Time series of tag frequency (Tag $1=$ blue, Tag 2 = orange). (b) Time series of strategy frequency for Tag 1 individuals $\left(C_{1}=\right.$ green, $I_{1}=$ purple, $E_{1}=$ red and $D_{1}=$ gray). (c) Time series of strategy frequency for Tag 2 individuals $\left(C_{2}=\right.$ green, $I_{2}=$ purple, $E_{2}=$ red and $D_{2}=$ gray).

spatial model for Region I when one tag excludes the other $(r=0.15)$. In this case, the aggregations of $I$-strategists form spontaneously and start to grow; the size of these aggregations eventually becomes so large that one tag completely overtakes the other (Fig. 4). Figures 5 and 6 show example

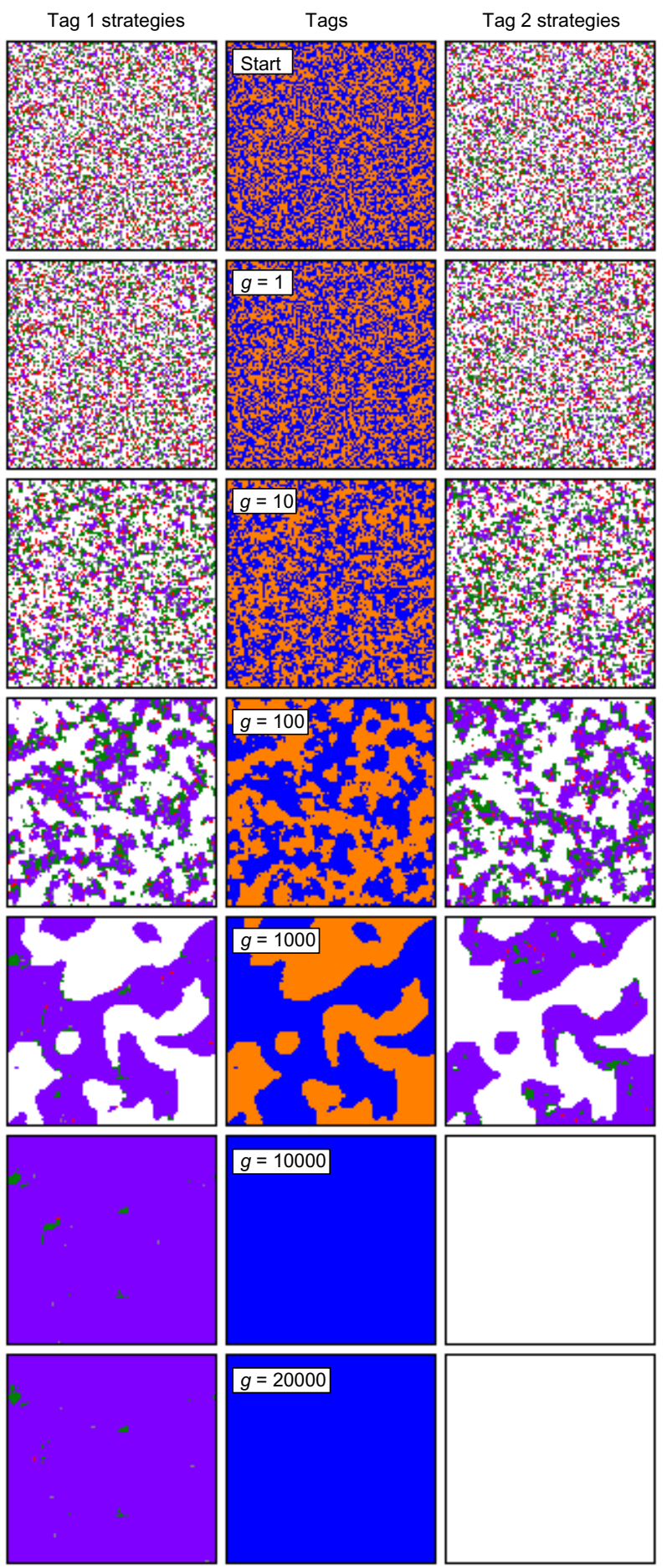

Fig. 4. Example arrangement of tags and strategies in the spatial, two-tag Snowdrift game for the same model run as in Fig. $3(r=0.15$ and $\mu=0)$, for the initial conditions (Start), and generations $g=1,10,100,1000,10000$ and 20000 . The middle column shows the tags' arrangement (Tag $1=$ blue, Tag 2 = orange), while the left and right columns show the strategies' arrangement for Tags 1 and 2, respectively $\left(C_{i}=\right.$ green, $I_{i}=$ purple, $E_{i}=$ red, $D_{i}=$ gray, where $i=1$ for Tag 1 and 2 for Tag 2). In Tag 1 (Tag 2) column, white space indicates an area that is occupied by Tag 2 (Tag 1) individuals. 


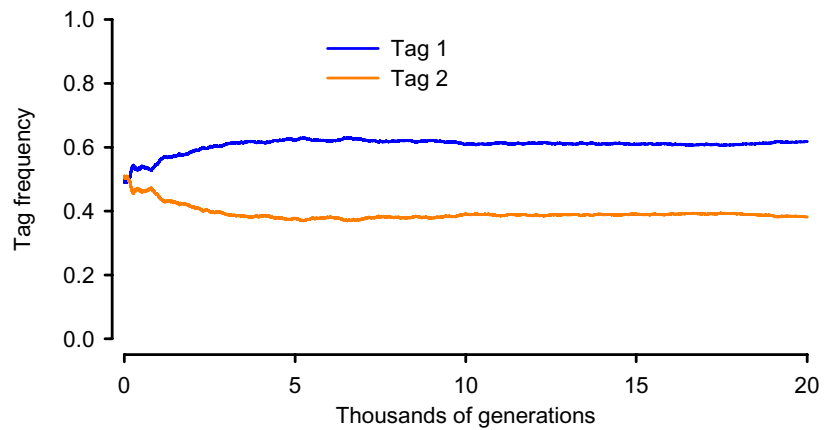

(a)

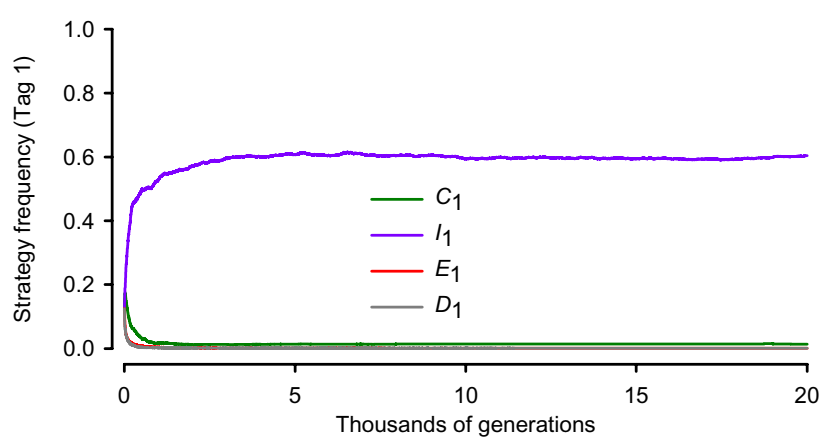

(b)

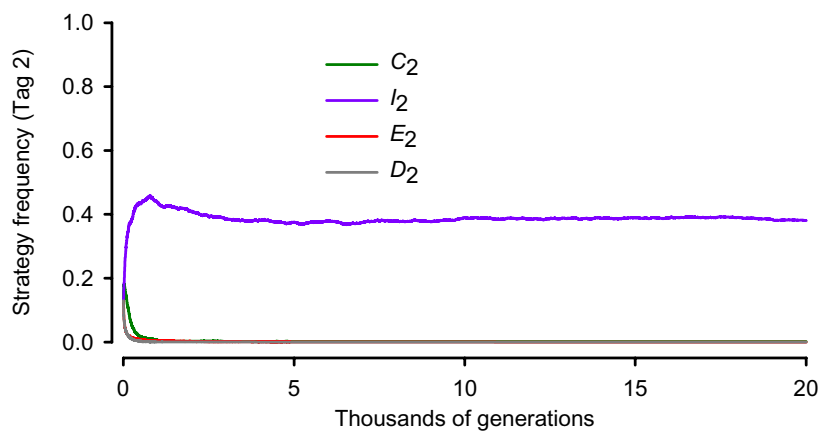

(c)

Fig. 5. Example dynamics for the spatial, two-tag Snowdrift game in the parameter region of $0<r<r_{1}$ (Region I; $r=$ 0.16 and $\mu=0$ ). (a) Time series of tag frequency (Tag $1=$ blue, Tag 2 = orange). (b) Time series of strategy frequency for Tag 1 individuals $\left(C_{1}=\right.$ green, $I_{1}=$ purple, $E_{1}=$ red and $D_{1}=$ gray). (c) Time series of strategy frequency for Tag 2 individuals $\left(C_{2}=\right.$ green, $I_{2}=$ purple, $E_{2}=$ red and $D_{2}=$ gray).

time-series and lattice snapshots for the spatial model for Region I when one tag fails to exclude the other $(r=0.16)$. In this example, chance brings the aggregations of $I_{1}$ and $I_{2}$ individuals into a standoff lattice configuration (e.g. a straight border region), resulting in their long-term quasicoexistence. Interestingly, in the larger $400 \times 400$ lattices with 50000 generations, tag exclusion is

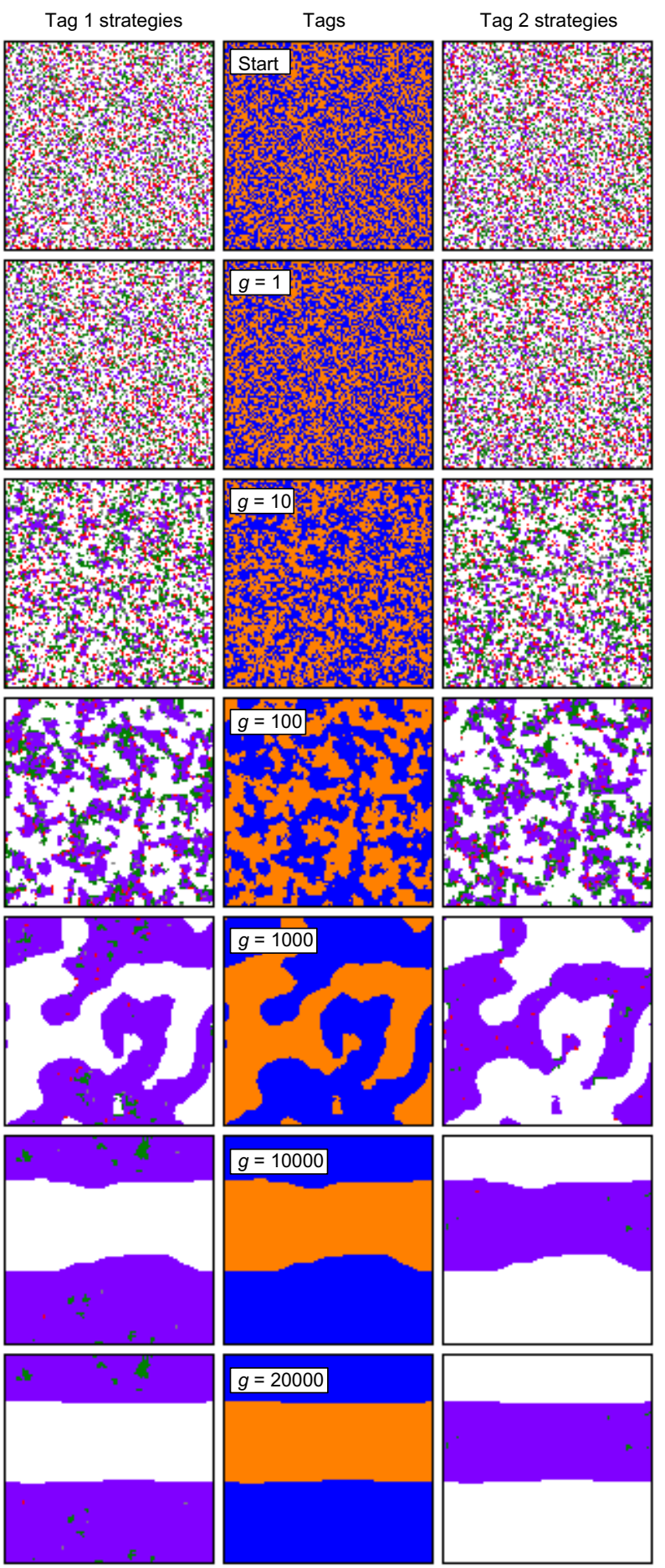

Fig. 6. Example arrangement of tags and strategies in the spatial, two-tag Snowdrift game for the same model run as in Fig. $5(r=0.16$ and $\mu=0)$, for the initial conditions (Start), and generations $g=1,10,100,1000,10000$ and 20000 . The middle column shows the tags' arrangement (Tag $1=$ blue, Tag 2 = orange), while the left and right columns show the strategies' arrangement for Tags 1 and 2, respectively $\left(C_{i}=\right.$ green, $I_{i}=$ purple, $E_{i}=$ red, $D_{i}=$ gray, where $i=1$ for Tag 1 and 2 for Tag 2). In Tag 1 (Tag 2) column, white space indicates an area that is occupied by Tag 2 (Tag 1) individuals. 
not observed in Region I [although large variation between tag frequencies did occasionally occur; Fig. 2(a)]. Nevertheless, and although it cannot be directly evaluated with the present data, I predict that given enough time, one tag's $I$-strategists would eventually overtake those of the other tag in this region as in Figs. 3 and 4, particularly when the cost-benefit ratio approaches its upper limit of $r_{1}$. This prediction stems from the fact that in a population predominantly composed of $I$-strategists, an "island" of $I$-strategists of one tag is vulnerable to a "sea" of $I$-strategists of the other tag, because the shape of the tag boundary ensures that those in the "sea" experience more mutual cooperation - and less mutual defection - than those in the "island". Thus, if one tag's I-strategists gain the upper hand by chance alone, then this trend is likely to be reinforced and even strengthened by the geometry of the spatial interactions. However, the resulting exclusion may take an exceptionally long time to occur because opposing tags can randomly prevail in different spatial areas of the population, or if standoff conditions emerge as in Figs. 5 and 6. The former is especially likely to be the case in large populations where distant spatial locations are not in phase with one another.

When $r_{1}<r<r_{2}$ (Region II), $D$-strategists abruptly begin to persist over the long term [Figs. 1(a)(iii) and 2(b)]. There are again two outcomes that are possible over 20000 model generations in $100 \times 100$ lattices; in the first outcome, one of the tags excludes the other, whereas in the second outcome, the tags coexist [Figs. 1(a)(i) and 2(a)]. Figures 7 and 8 show example time-series and lattice snapshots for the spatial model for Region II when one tag excludes the other $(r=0.28)$. For this outcome, same-tagged $D$ - and $I$-strategists form mixed-strategy aggregations (Fig. 8), similar to the dendritic structures exhibited by cooperators and defectors in the non-tag-based Snowdrift models of Hauert and Doebeli [2004] and Doebeli and Hauert [2005], that increase in size until one tag overtakes the other. In contrast, Figs. 9 and 10 show example time-series and lattice snapshots for the spatial model for Region II $(r=0.31)$, in which the two tags reach a standoff configuration that allows for long-term quasi-coexistence. As with Region I, this second outcome in Region II is likely a transitory (albeit potentially long-lived) phenomenon; however unlike Region I, tag exclusion can be observed even in the larger lattices [Fig. 2(a)].

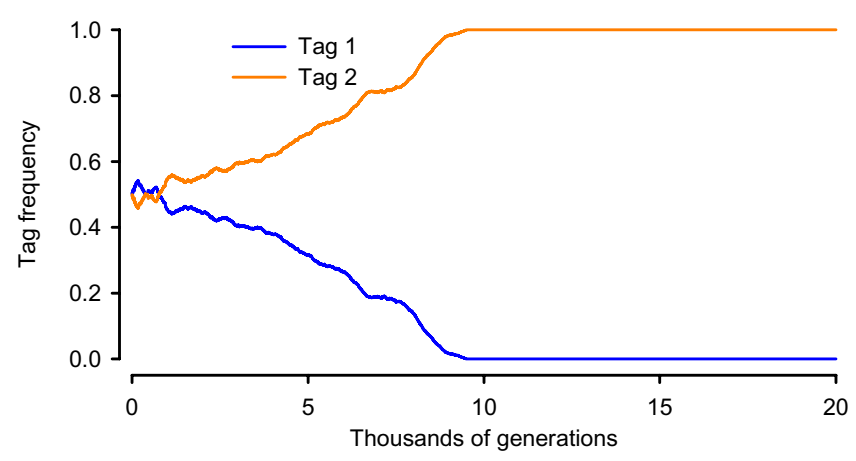

(a)

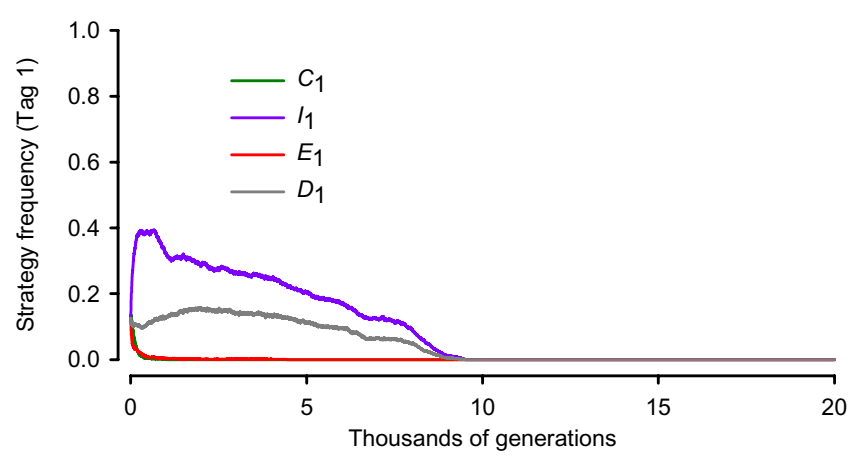

(b)

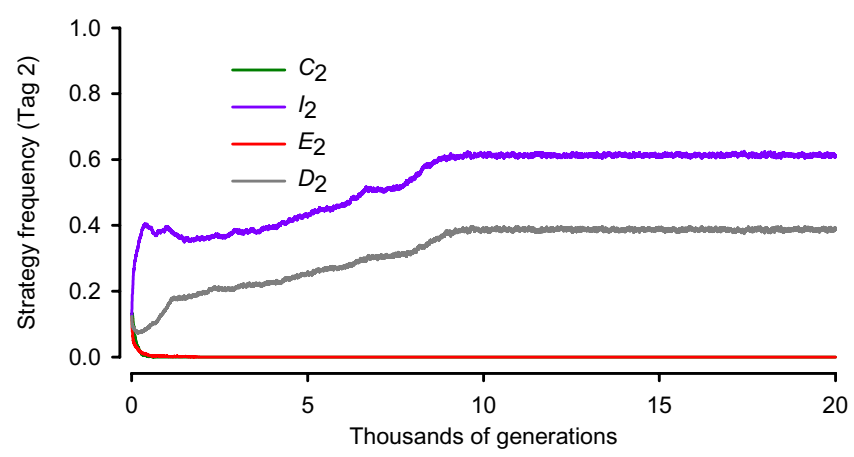

(c)

Fig. 7. Example dynamics for the spatial, two-tag Snowdrift game in the parameter region of $r_{1}<r<r_{2}$ (Region II; $r=$ 0.28 and $\mu=0$ ). (a) Time series of tag frequency (Tag $1=$ blue, Tag $2=$ orange). (b) Time series of strategy frequency for Tag 1 individuals $\left(C_{1}=\right.$ green, $I_{1}=$ purple, $E_{1}=$ red and $D_{1}=$ gray). (c) Time series of strategy frequency for Tag 2 individuals $\left(C_{2}=\right.$ green, $I_{2}=$ purple, $E_{2}=$ red and $D_{2}=$ gray).

In Region III $\left(r_{2}<r<r_{3}\right)$ and Region V $(r>$ $r_{4}$ ), the two tags - and two of the strategies, $I$ and $D$ - coexist in the long term [Figs. 1(a)(i), 1(a)(iii), 2(a) and 2(b)]. Figures 11 and 12 show example time-series and lattice snapshots for the spatial model for Region III $(r=0.42)$; Figs. 13 and 14 show example time-series and lattice snapshots for 


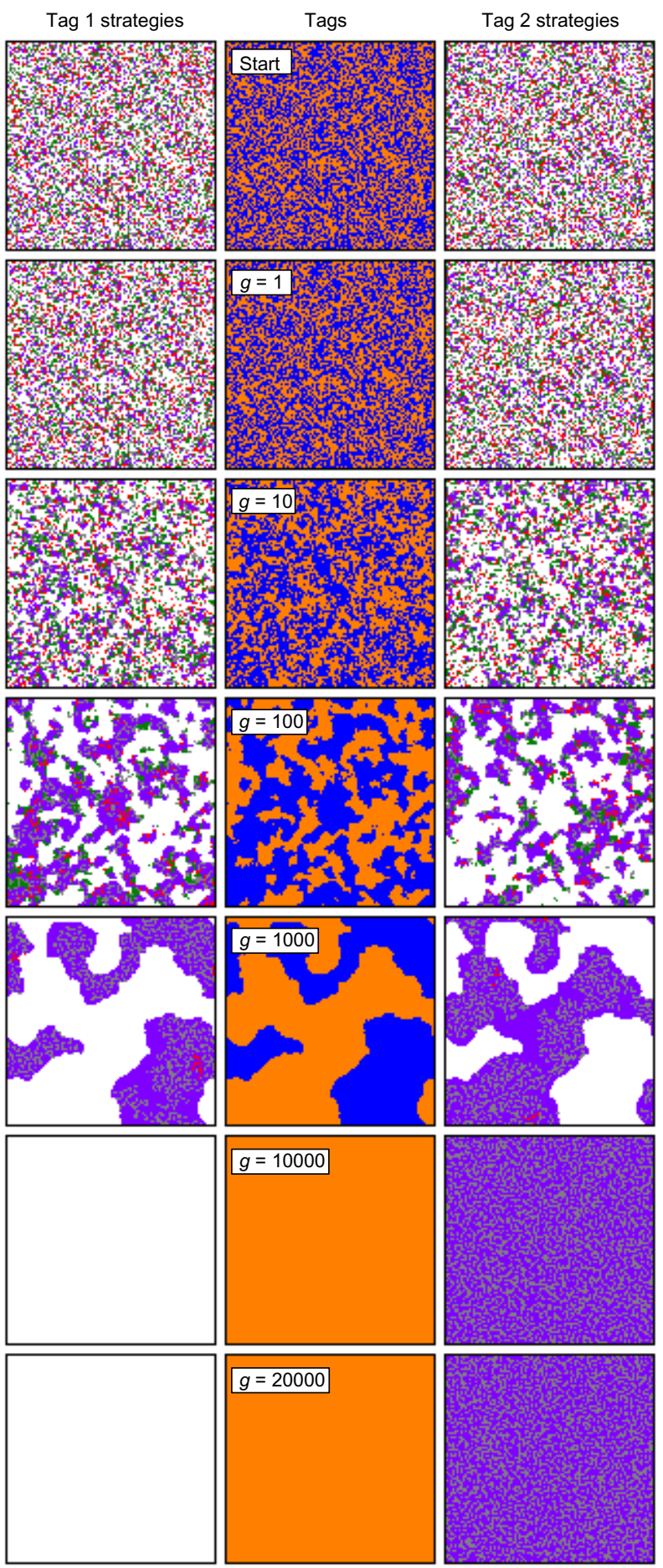

Fig. 8. Example arrangement of tags and strategies in the spatial, two-tag Snowdrift game for the same model run as in Fig. $7(r=0.28$ and $\mu=0)$, for the initial conditions (Start), and generations $g=1,10,100,1000,10000$ and 20000 . The middle column shows the tags' arrangement (Tag $1=$ blue, Tag 2 = orange), while the left and right columns show the strategies' arrangement for Tags 1 and 2, respectively $\left(C_{i}=\right.$ green, $I_{i}=$ purple, $E_{i}=$ red, $D_{i}=$ gray, where $i=1$ for Tag 1 and 2 for Tag 2). In Tag 1 (Tag 2) column, white space indicates an area that is occupied by Tag 2 (Tag 1) individuals.

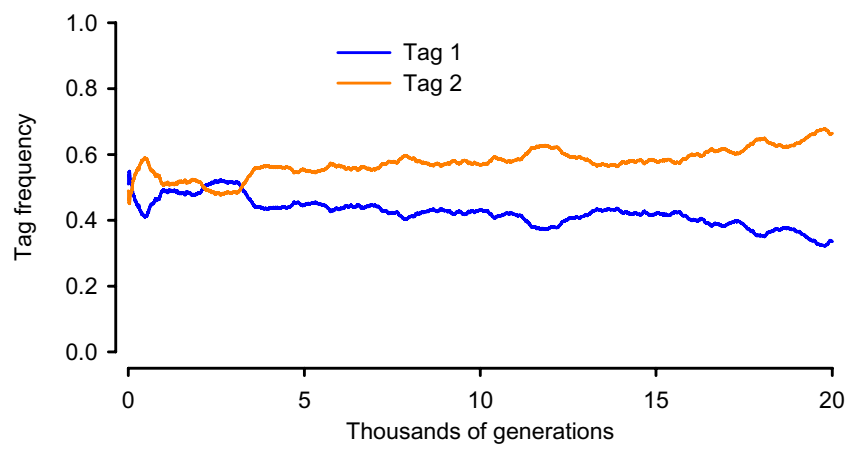

(a)

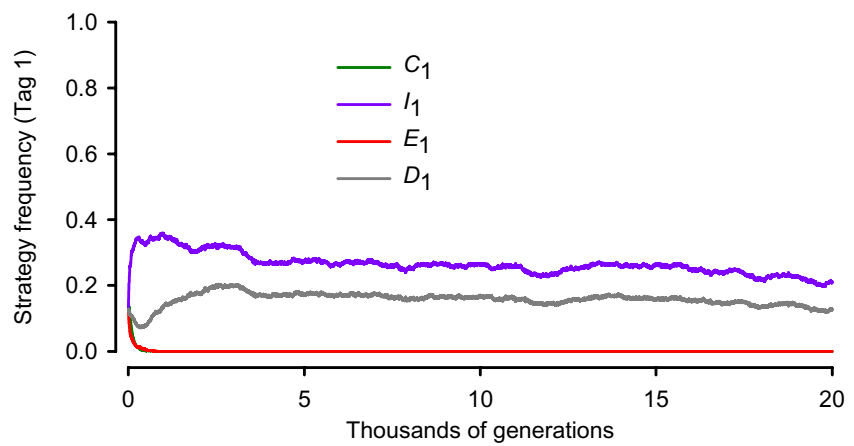

(b)

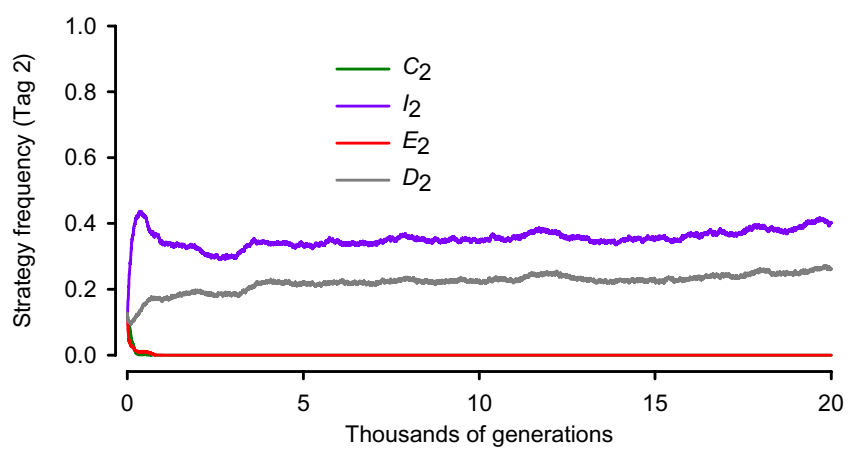

(c)

Fig. 9. Example dynamics for the spatial, two-tag Snowdrift game in the parameter region of $r_{1}<r<r_{2}$ (Region II; $r=$ 0.31 and $\mu=0$ ). (a) Time series of tag frequency (Tag $1=$ blue, Tag 2 = orange). (b) Time series of strategy frequency for Tag 1 individuals $\left(C_{1}=\right.$ green, $I_{1}=$ purple, $E_{1}=$ red and $D_{1}=$ gray). (c) Time series of strategy frequency for Tag 2 individuals $\left(C_{2}=\right.$ green, $I_{2}=$ purple, $E_{2}=$ red and $D_{2}=$ gray).

the spatial model for Region $\mathrm{V}(r=0.80)$. Note that unlike Regions I and II, tags and strategies in Regions III and V exhibit cyclic dynamics (Figs. 11 and 13). Figure 15(a) shows the period of cyclic dynamics of Tag 1 , in the region where such cycles are detectable by autocorrelation analysis (based on time series from $L^{2}=10000$ cell lattices over 20000 


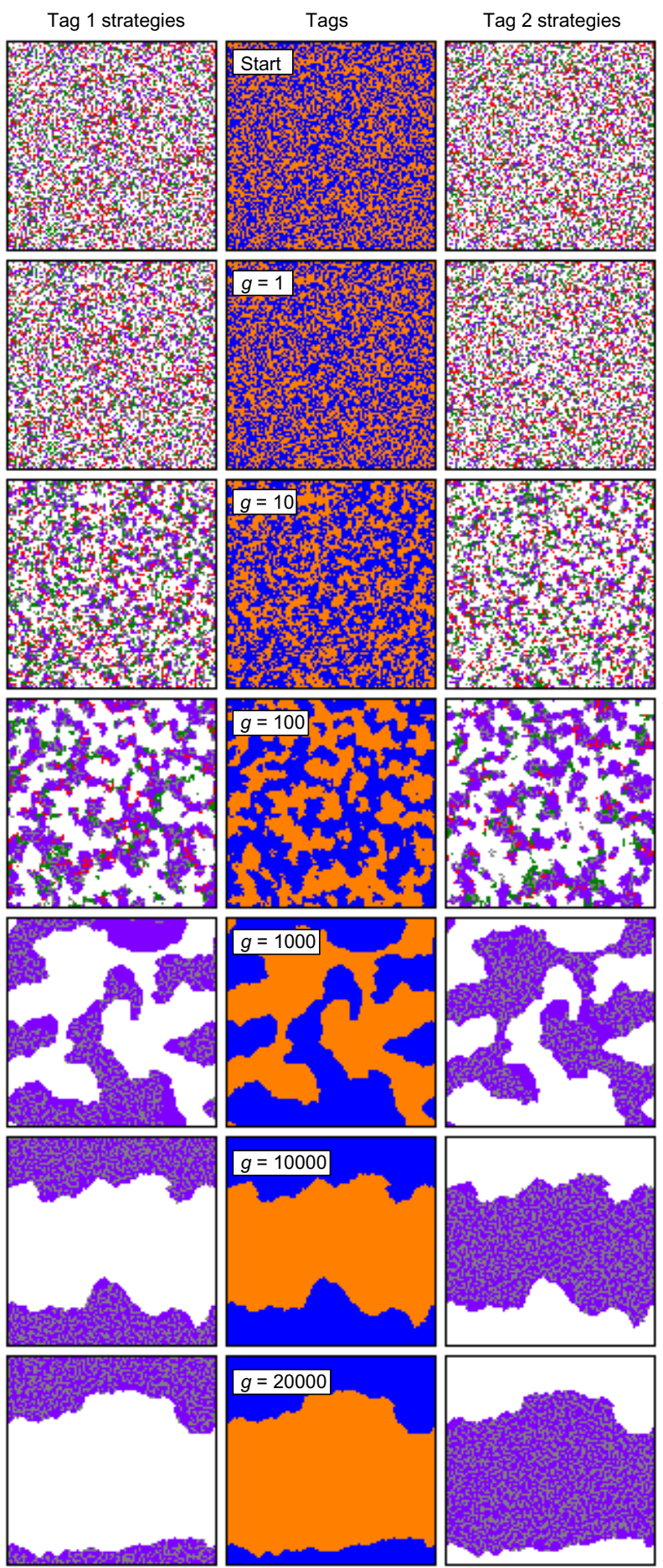

Fig. 10. Example arrangement of tags and strategies in the spatial, two-tag Snowdrift game for the same model run as in Fig. $9(r=0.31$ and $\mu=0)$, for the initial conditions (Start), and generations $g=1,10,100,1000,10000$ and 20000 . The middle column shows the tags' arrangement (Tag $1=$ blue, Tag $2=$ orange), while the left and right columns show the strategies' arrangement for Tags 1 and 2, respectively $\left(C_{i}=\right.$ green, $I_{i}=$ purple, $E_{i}=$ red, $D_{i}=$ gray, where $i=1$ for Tag 1 and 2 for Tag 2). In Tag 1 (Tag 2) column, white space indicates an area that is occupied by Tag 2 (Tag 1) individuals.

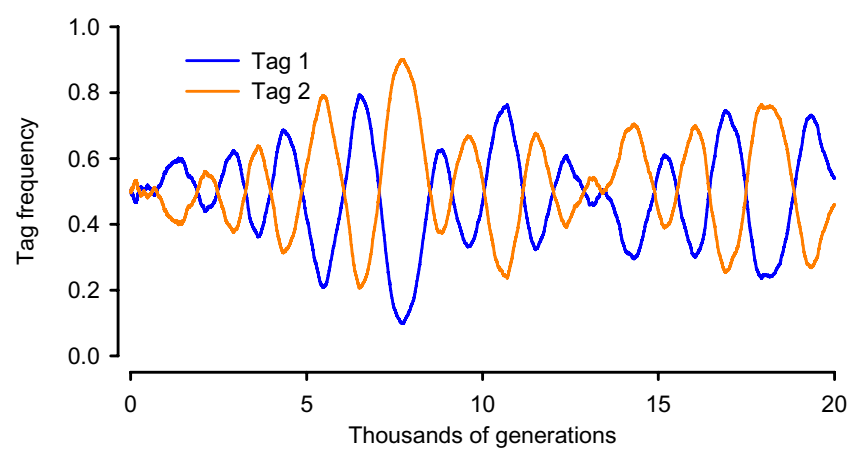

(a)

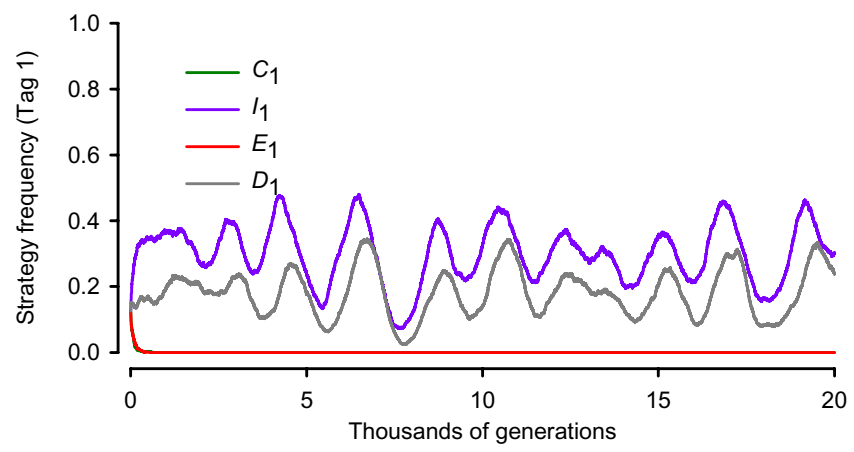

(b)

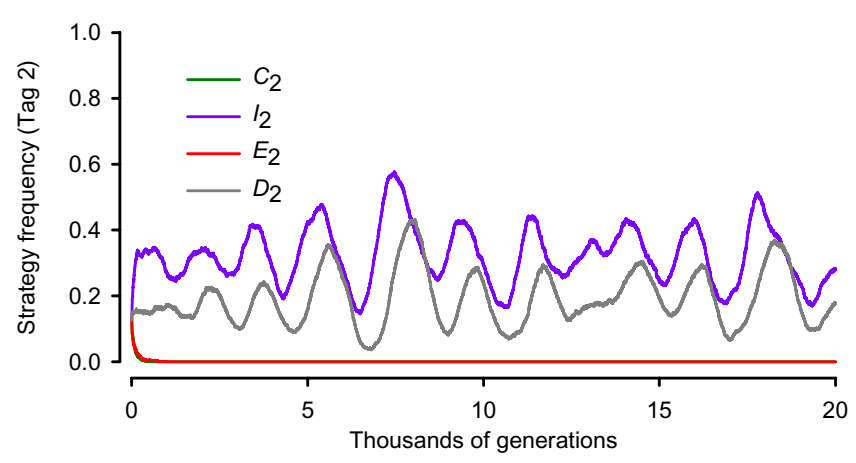

(c)

Fig. 11. Example dynamics for the spatial, two-tag Snowdrift game in the parameter region of $r_{2}<r<r_{3}$ (Region III; $r=0.42$ and $\mu=0$ ). (a) Time series of tag frequency (Tag $1=$ blue, Tag 2 = orange). (b) Time series of strategy frequency for Tag 1 individuals $\left(C_{1}=\right.$ green, $I_{1}=$ purple, $E_{1}=$ red and $D_{1}=$ gray). (c) Time series of strategy frequency for Tag 2 individuals $\left(C_{2}=\right.$ green, $I_{2}=$ purple, $E_{2}=\operatorname{red}$ and $D_{2}=$ gray).

generations). Note that the region where cycles are detectable directly corresponds with Regions III-V . Within this same region, cross-correlation analysis shows that the strategy cycles progress as follows [the starting point in this description is, of course, arbitrary; $C$ - and $E$-strategists are omitted here due 


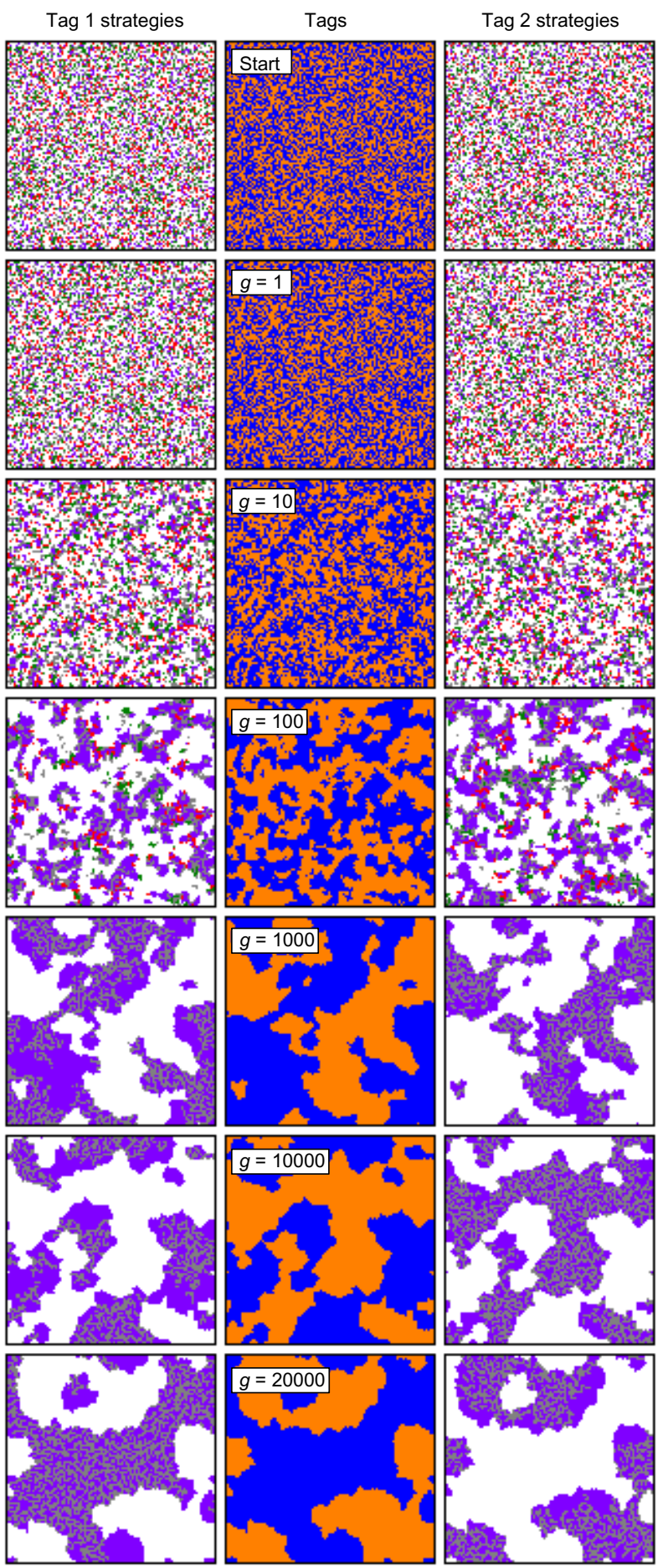

Fig. 12. Example arrangement of tags and strategies in the spatial, two-tag Snowdrift game for the same model run as in Fig. $11(r=0.42$ and $\mu=0)$, for the initial conditions (Start), and generations $g=1,10,100,1000,10000$ and 20000 . The middle column shows the tags' arrangement (Tag $1=$ blue, Tag 2 =orange), while the left and right columns show the strategies' arrangement for Tags 1 and 2, respectively $\left(C_{i}=\right.$ green, $I_{i}=$ purple, $E_{i}=$ red, $D_{i}=$ gray, where $i=1$ for Tag 1 and 2 for Tag 2). In Tag 1 (Tag 2) column, white space indicates an area that is occupied by Tag 2 (Tag 1) individuals.

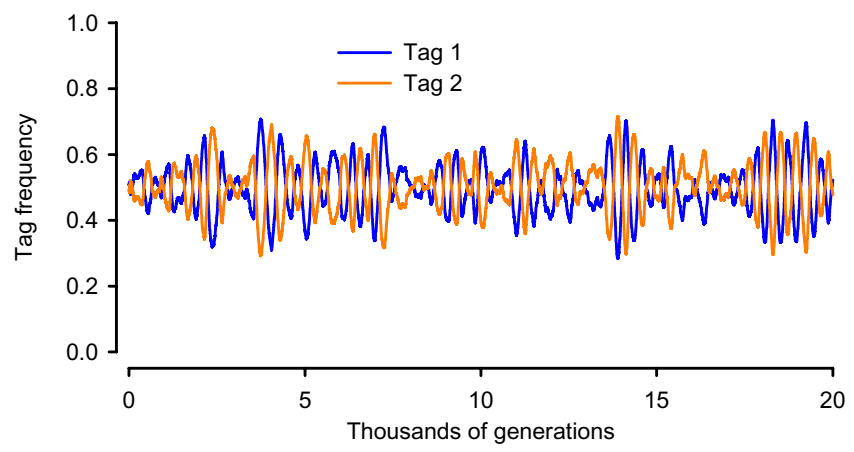

(a)

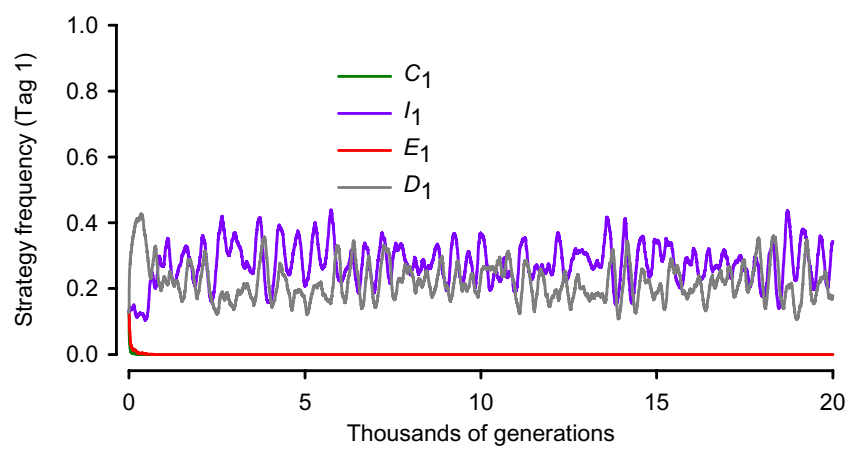

(b)

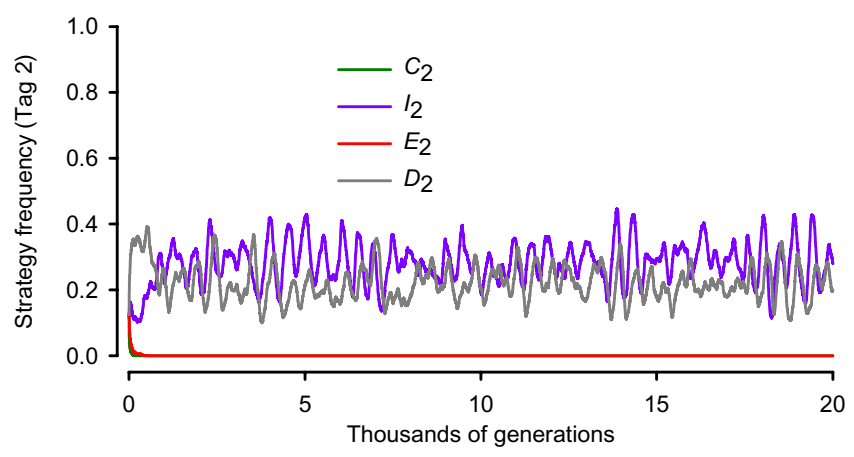

(c)

Fig. 13. Example dynamics for the spatial, two-tag Snowdrift game in the parameter region of $r>r_{4}$ (Region $\mathrm{V}$; $r=0.80$ and $\mu=0$ ). (a) Time series of tag frequency (Tag $1=$ blue, Tag $2=$ orange). (b) Time series of strategy frequency for Tag 1 individuals $\left(C_{1}=\right.$ green, $I_{1}=$ purple, $E_{1}=$ red and $D_{1}=$ gray). (c) Time series of strategy frequency for Tag 2 individuals $\left(C_{2}=\right.$ green, $I_{2}=$ purple, $E_{2}=$ red and $D_{2}=$ gray).

to low abundance; Fig. 15(b)]: first, $I_{1}$ peaks, with $D_{1}$ lagging a partial cycle behind. Then, strategy $I_{2}$ peaks, one-half cycle out of phase with $I_{1}$, again with $D_{2}$ lagging a partial cycle behind (and onehalf cycle behind $D_{1}$ ). This order of cyclic replacement occurs due to nontransitive spatial invasibility 


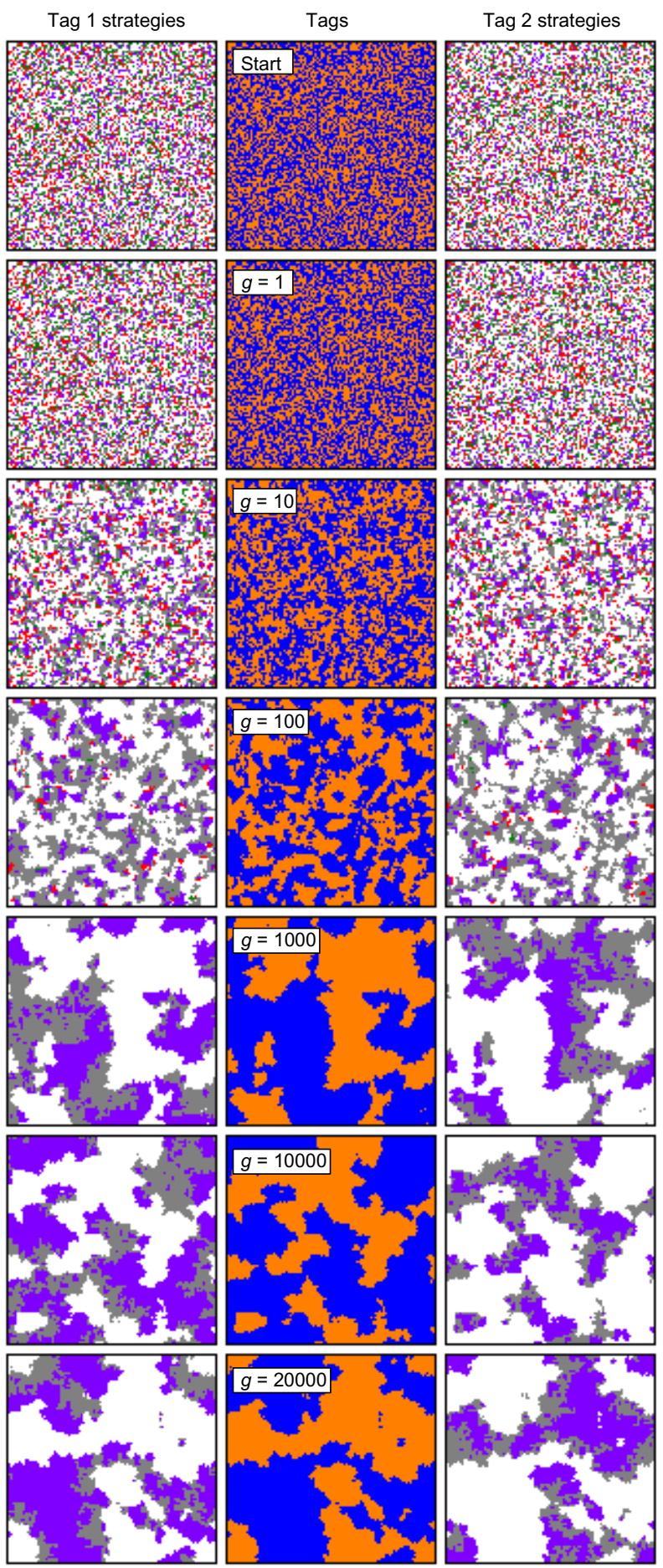

Fig. 14. Example arrangement of tags and strategies in the spatial, two-tag Snowdrift game for the same model run as in Fig. $13(r=0.80$ and $\mu=0)$, for the initial conditions (Start), and generations $g=1,10,100,1000,10000$ and 20000 . The middle column shows the tags' arrangement (Tag $1=$ blue, Tag 2 = orange), while the left and right columns show the strategies' arrangement for Tags 1 and 2, respectively $\left(C_{i}=\right.$ green, $I_{i}=$ purple, $E_{i}=$ red, $D_{i}=$ gray, where $i=1$ for Tag 1 and 2 for Tag 2). In Tag 1 (Tag 2) column, white space indicates an area that is occupied by Tag 2 (Tag 1) individuals.

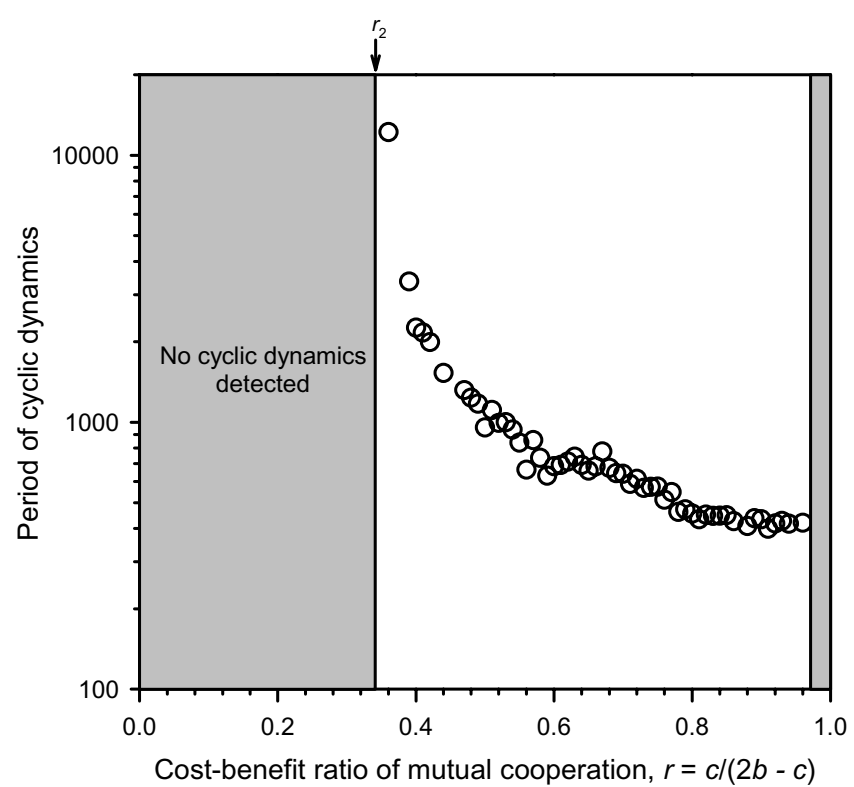

(a)

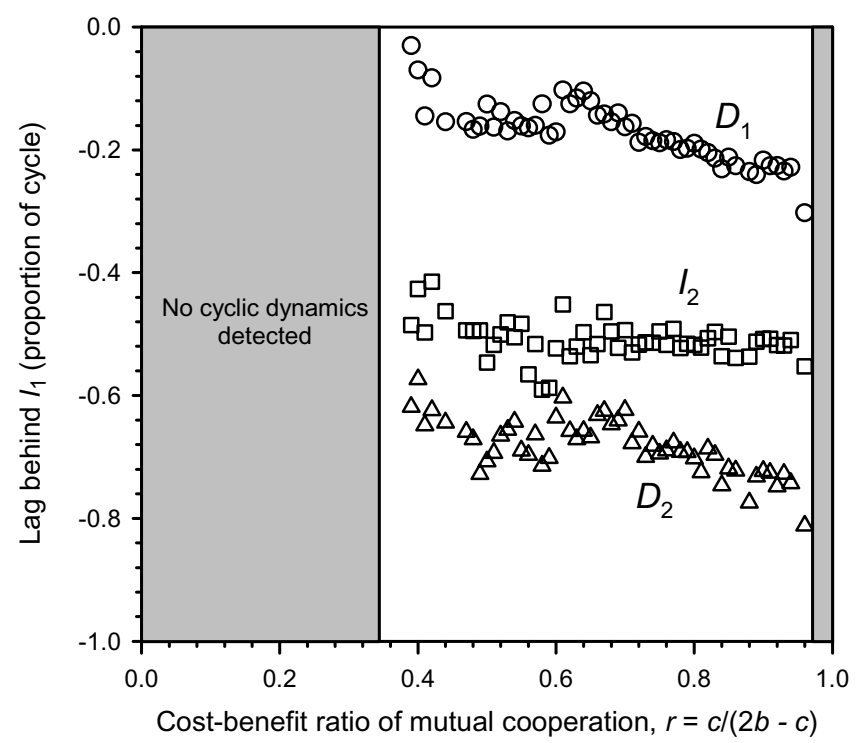

(b)

Fig. 15. (a) Period of cyclic dynamics (in generations) based on temporal autocorrelation of Tag 1 frequencies for the spatial, two-tag Snowdrift game for parameter values above $r_{2}$ (cyclic dynamics were not detected elsewhere). (b) The lag with which cycles of $D_{1}$ (circles), $I_{2}$ (squares) and $D_{2}$ (triangles) trail cycles of $I_{1}$ for the same region of $r$ as in (a).

[Laird, 2011] whereby $I_{1}$ invades patches occupied by mixtures of $I_{2}$ and $D_{2}$, while $I_{2}$ invades patches occupied by mixtures of $I_{1}$ and $D_{1}$, yet $D_{1}$ invades $I_{1}$ (reconstituting mixtures of $I_{1}$ and $D_{1}$ ) and $D_{2}$ invades $I_{2}$ (reconstituting mixtures of $I_{2}$ and $D_{2}$ ). The spatial signature of this nontransitivity can be 


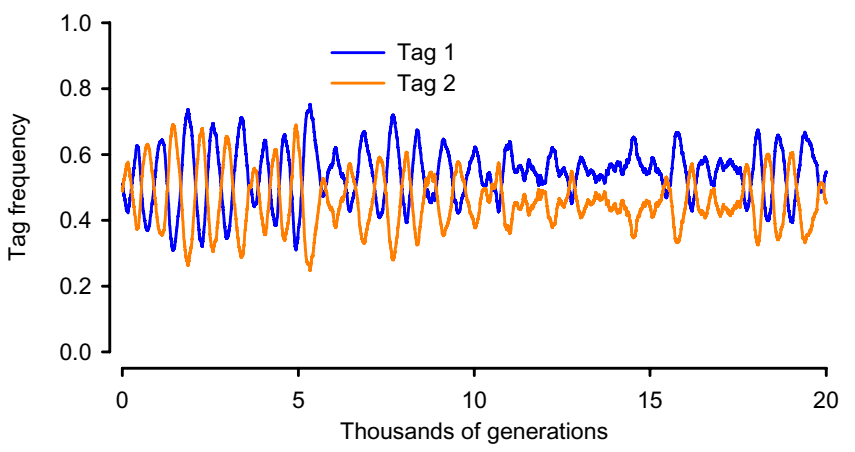

(a)

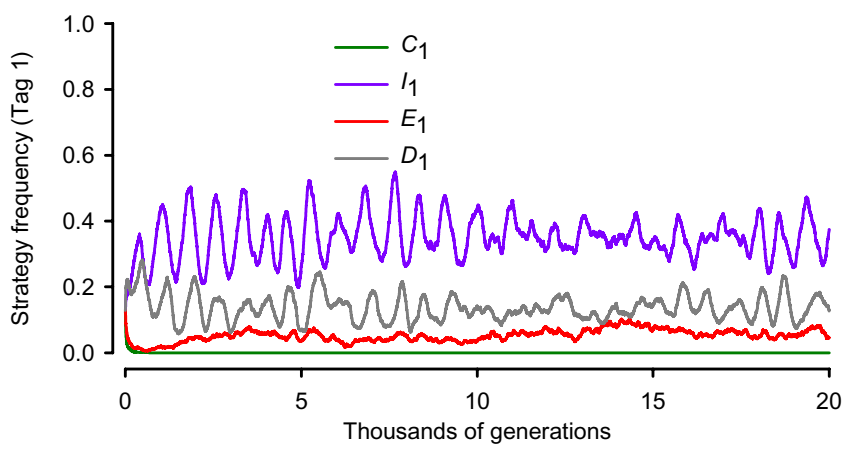

(b)

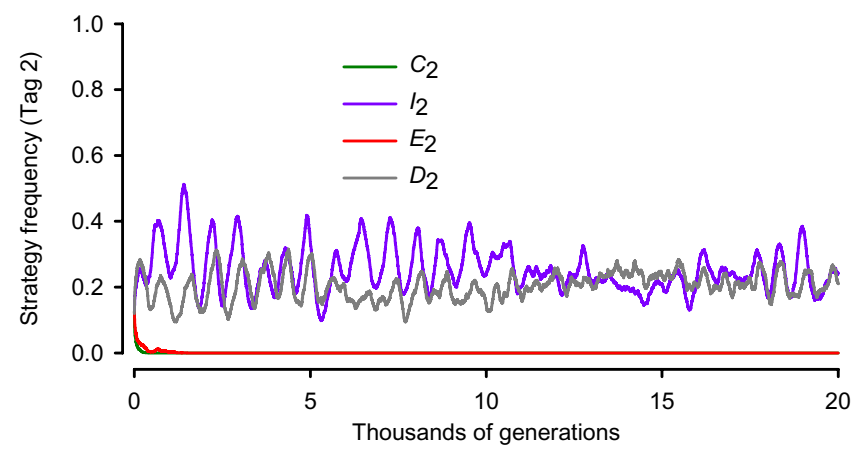

(c)

Fig. 16. Example dynamics for the spatial, two-tag Snowdrift game in the parameter region of $r_{3}<r<r_{4}$ (Region IV; $r=0.60$ and $\mu=0$ ). (a) Time series of tag frequency (Tag $1=$ blue, Tag $2=$ orange). (b) Time series of strategy frequency for Tag 1 individuals $\left(C_{1}=\right.$ green, $I_{1}=$ purple, $E_{1}=$ red and $D_{1}=$ gray). (c) Time series of strategy frequency for Tag 2 individuals $\left(C_{2}=\right.$ green, $I_{2}=$ purple, $E_{2}=$ red and $D_{2}=$ gray).

seen in Fig. 12, where, for example, monocultures of $I_{1}$ and $I_{2}$ are only present in areas where they are invading territory currently held by mixtures of $I_{2}$ and $D_{2}$, and $I_{1}$ and $D_{1}$, respectively.

Other than at low $r$-values where they are present in minute numbers, Region IV $\left(r_{3}<r<r_{4}\right)$

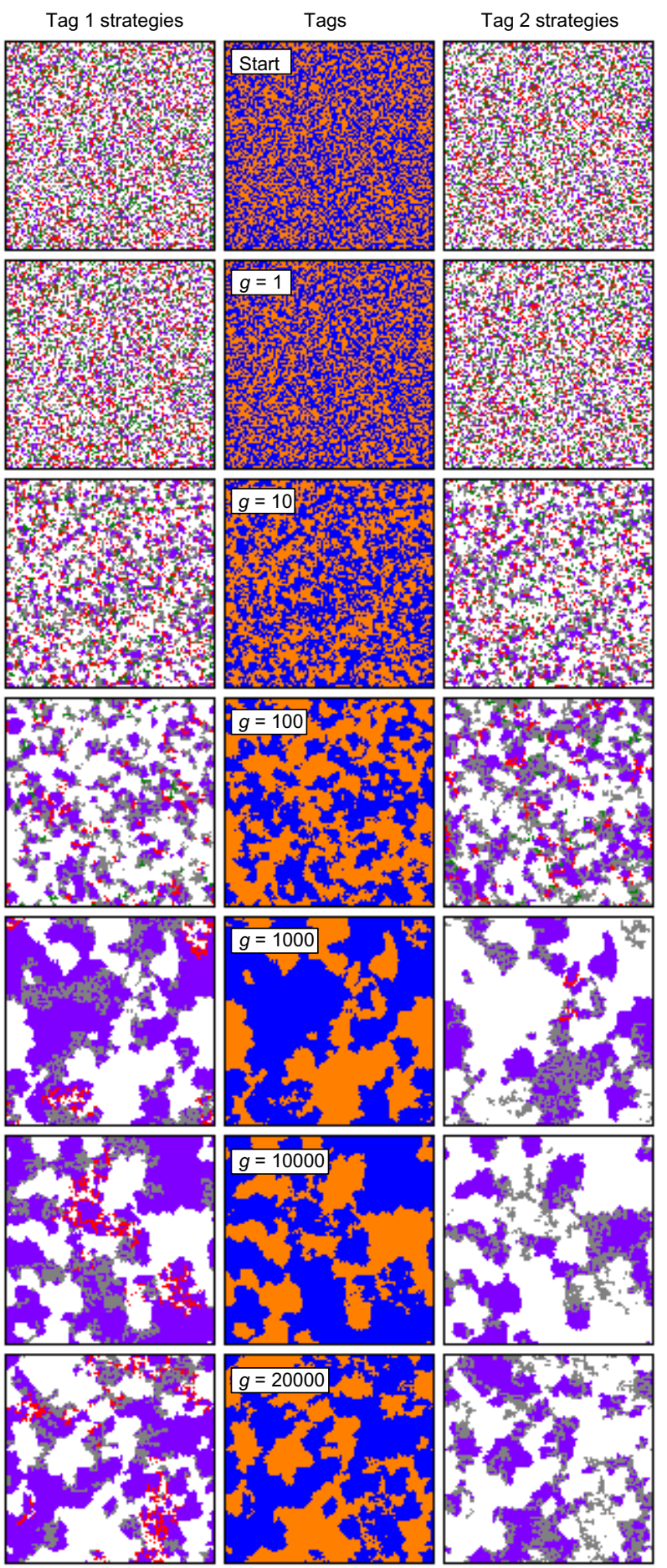

Fig. 17. Example arrangement of tags and strategies in the spatial, two-tag Snowdrift game for the same model run as in Fig. $16(r=0.60$ and $\mu=0)$, for the initial conditions (Start), and generations $g=1,10,100,1000,10000$ and 20000 . The middle column shows the tags' arrangement (Tag $1=$ blue, Tag 2 = orange), while the left and right columns show the strategies' arrangement for Tags 1 and 2, respectively $\left(C_{i}=\right.$ green, $I_{i}=$ purple, $E_{i}=$ red, $D_{i}=$ gray, where $i=1$ for Tag 1 and 2 for Tag 2). In Tag 1 (Tag 2) column, white space indicates an area that is occupied by Tag 2 (Tag 1) individuals. 


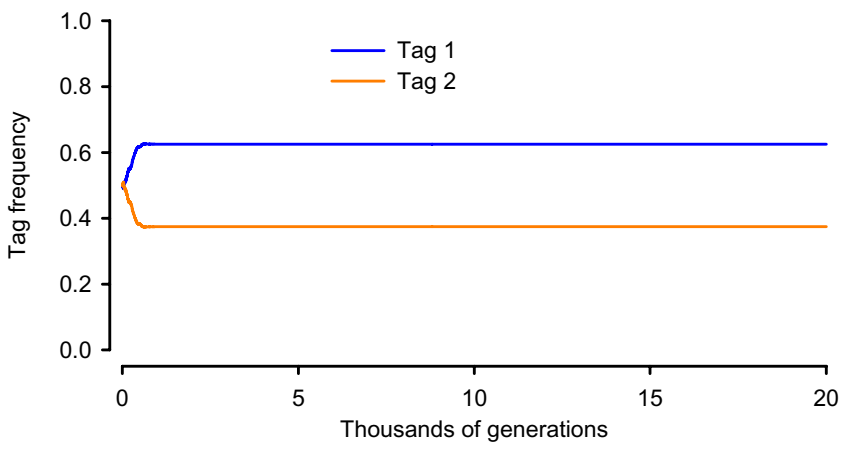

(a)

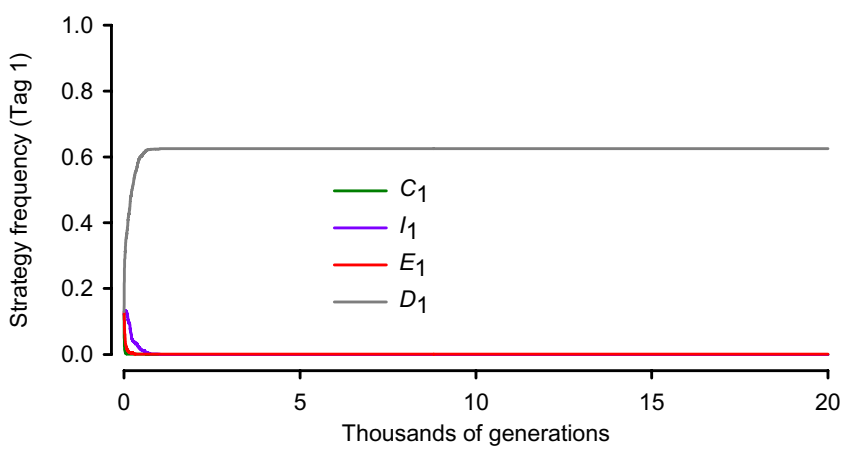

(b)

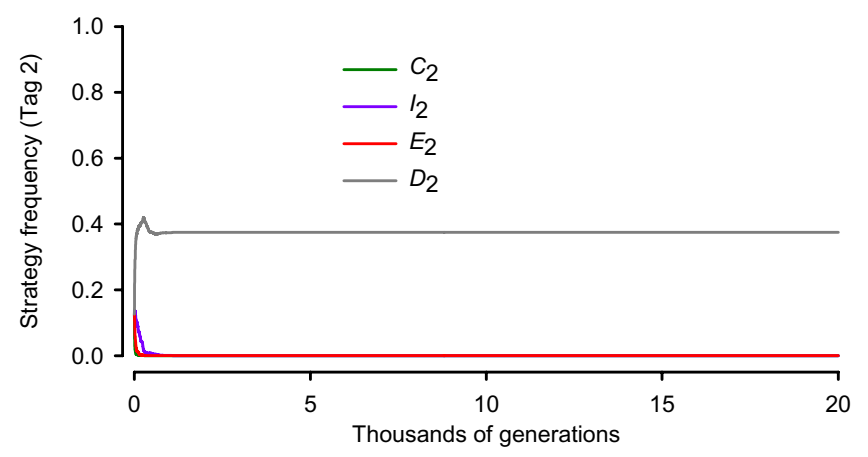

(c)

Fig. 18. Example dynamics for the spatial, two-tag Snowdrift game when $r=0.98$ and $\mu=0$. (a) Time series of tag frequency (Tag $1=$ blue, Tag 2 = orange). (b) Time series of strategy frequency for Tag 1 individuals $\left(C_{1}=\right.$ green, $I_{1}=$ purple, $E_{1}=$ red and $D_{1}=$ gray). (c) Time series of strategy frequency for Tag 2 individuals $\left(C_{2}=\right.$ green, $I_{2}=$ purple, $E_{2}=$ red and $D_{2}=$ gray).

is the only region where $E$-strategists reliably persist [albeit still at very low frequencies compared to $I$ and $D$; Figs. 1(a)(iii) and 2(b)]. Figures 16 and 17 show example time-series and lattice snapshots for the spatial model for Region IV $(r=0.60)$. Thus, in Region IV there is a balance between interactions that are favorable to $E$-strategists (e.g. with

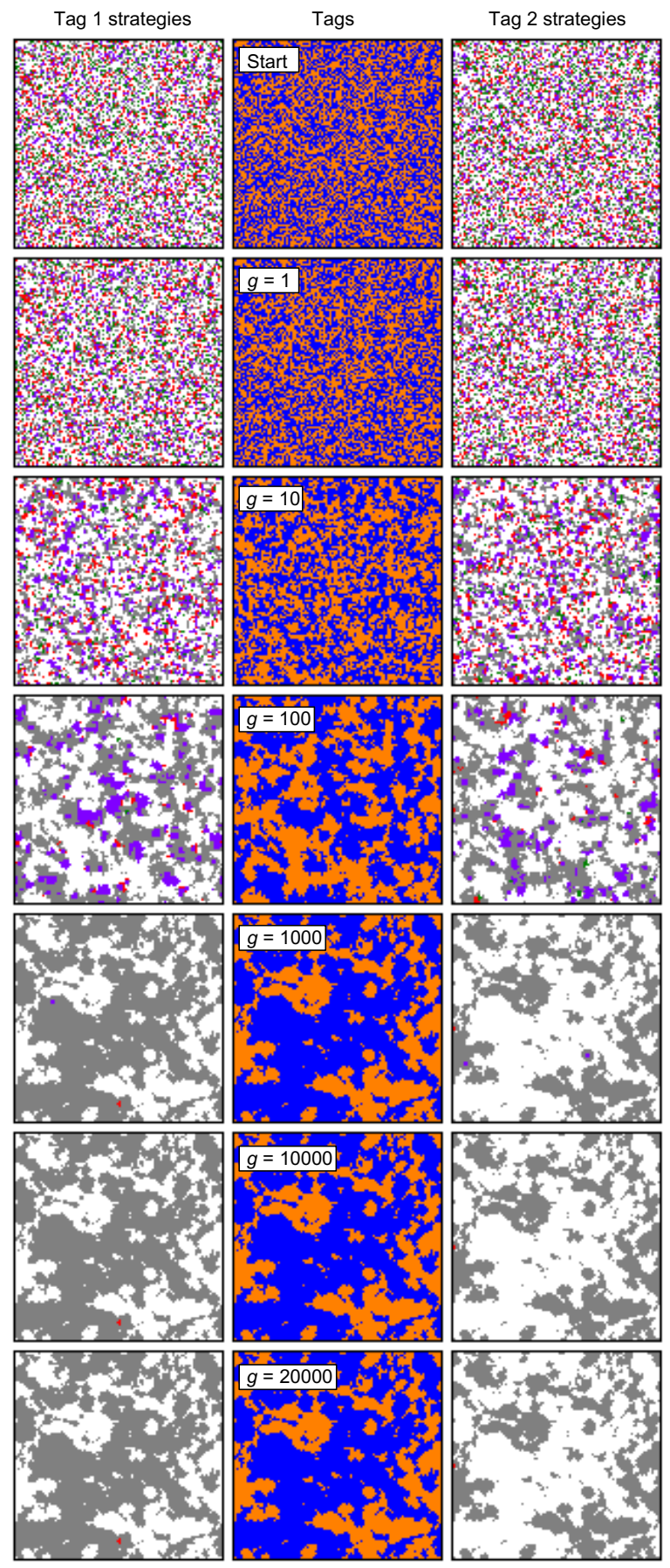

Fig. 19. Example arrangement of tags and strategies in the spatial, two-tag Snowdrift game for the same model run as in Fig. $18(r=0.98$ and $\mu=0)$, for the initial conditions (Start), and generations $g=1,10,100,1000,10000$ and 20000 . The middle column shows the tags' arrangement (Tag $1=$ blue, Tag 2 = orange), while the left and right columns show the strategies' arrangement for Tags 1 and 2, respectively $\left(C_{i}=\right.$ green, $I_{i}=$ purple, $E_{i}=$ red, $D_{i}=$ gray, where $i=1$ for Tag 1 and 2 for Tag 2). In Tag 1 (Tag 2) column, white space indicates an area that is occupied by Tag 2 (Tag 1) individuals. Note that the lattice reaches a deadlocked state, which occurs when all the individuals have the same payoff and there is no mutation. 
(a)

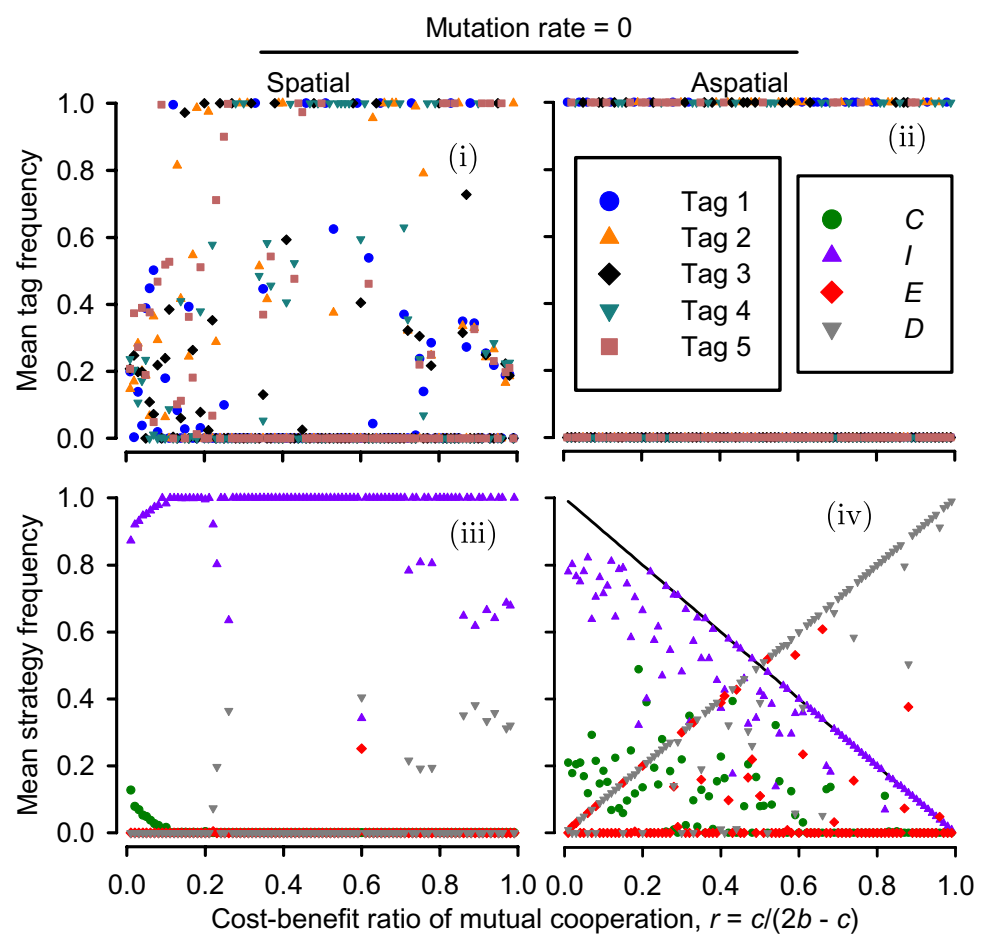

(b)

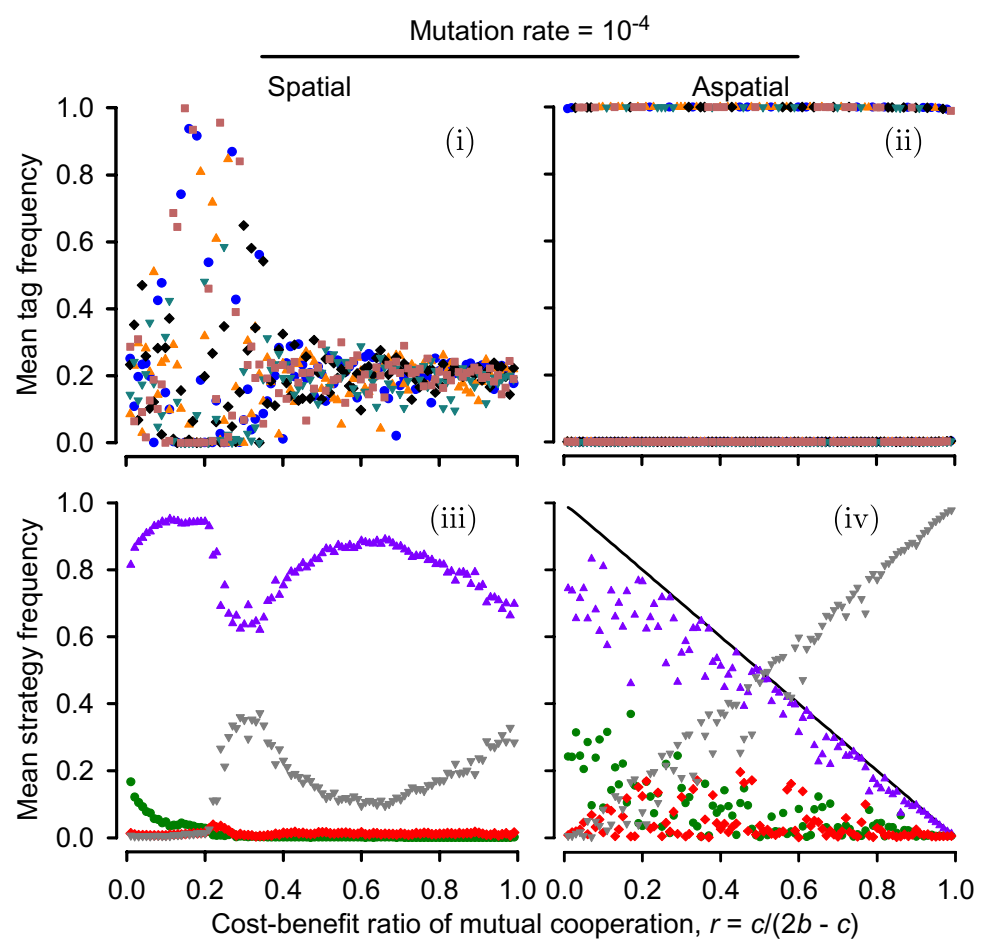

Fig. 20. Average tag [parts (i) and (ii)] and strategy relative abundances [parts (iii) and (iv)] for 5000 generations, starting after generation 15000, for the spatial [left column; parts (i) and (iii)] and aspatial [right column; parts (ii) and (iv)] five-tag Snowdrift game. Both the spatial and aspatial versions of the model had a fixed population size of 10000 . Initially, every individual was given a random tag and strategy. Colors: Tag $1=$ blue circles, Tag $2=$ orange upward-triangles, Tag $3=$ black diamonds, Tag $4=$ turquoise downward-triangles, Tag $5=$ rose squares, unconditional cooperators $(C)=$ green circles, intratag cooperators $(I)=$ purple upward-triangles, extra-tag cooperators $(E)=$ red diamonds, unconditional defectors $(D)=$ gray downward-triangles. Mutation rates: (a) $\mu=0$ (no mutation), (b) $\mu=10^{-4}$ and (c) $\mu=10^{-2}$. A critical value of $r$ is indicated by arrows in (c)(ii) and (c)(iv). At values of $r$ below this critical value, unconditional cooperators (i.e. $C$-strategists) abruptly become the most common strategy, allowing for the coexistence of all five tags at equal frequencies. 
(c)
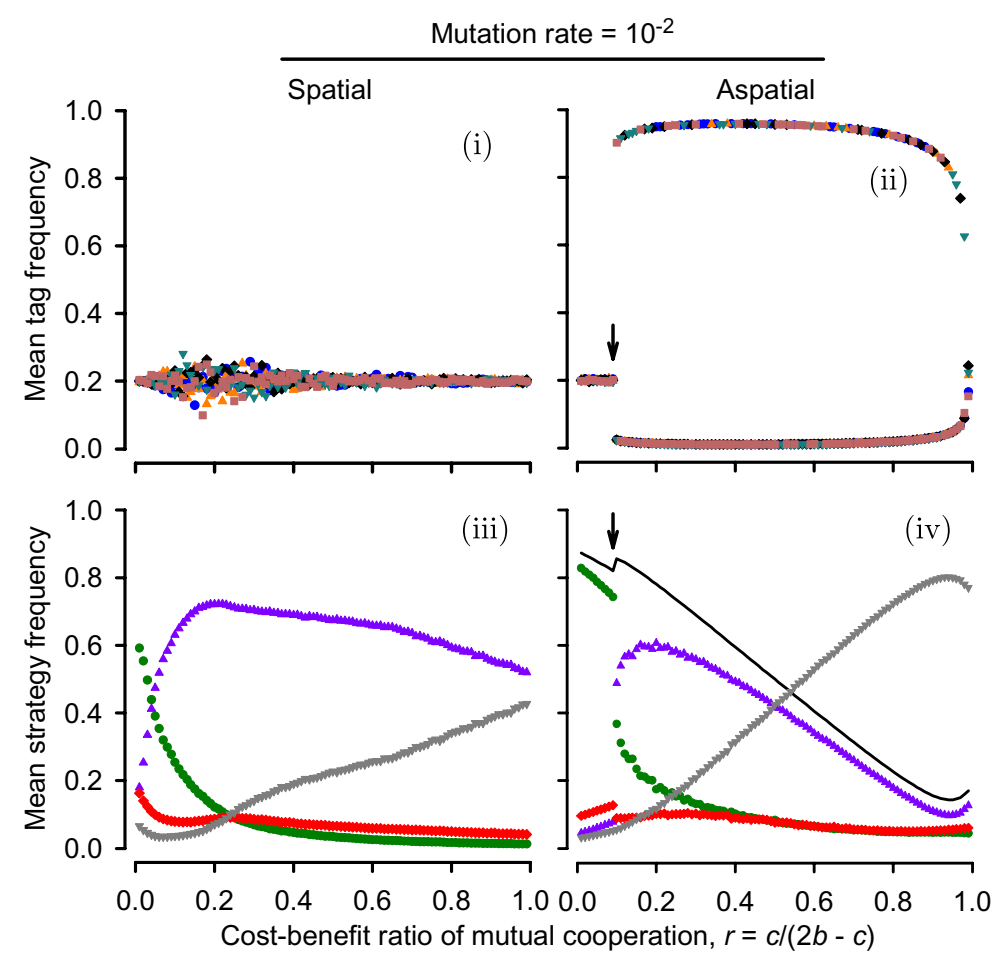

Fig. 20. (Continued)

same-tagged $I$-strategists) and interactions that are unfavorable to $E$-strategists (e.g. with differenttagged $D$-strategists), allowing $E$-strategists to simultaneously exploit and be exploited at approximately the same rate.

At very high values of $r$, close to the limit of the Snowdrift game $(r<1)$, $D$-strategists sometimes drive $I$-strategists extinct in finite, spatial populations [Figs. 1(a)(iii) and 2(b)]. For example, Figs. 18 and 19 show example time-series and lattice snapshots for $r=0.98$ (for $100 \times 100$ lattices over 20000 generations). This switch from $I-D$ coexistence to $D$ domination does not appear to be a true phase transition, but rather an artefact of finite populations; indeed it is only rarely observed in the larger lattices [contrast Fig. 1(a)(iii) with Fig. 2(b)].

\subsection{Simulation results in the presence of mutation: Aspatial and spatial two-tag models}

When a small amount of mutation is added to the models [e.g. one mutant per generation, on average $\left(\mu=10^{-4}\right.$ in a $100 \times 100$ lattice $)$; Fig. $\left.1(\mathrm{~b})\right]$, the same qualitative results apply, albeit with the boundaries between regions softened, and, in some cases, a lack of complete tag or strategy exclusion due to mutation-selection balance, which allows weaker strategies to persist at low frequencies. On the other hand, when the mutation rate is quite high [e.g. 100 mutants per generation, on average $\left(\mu=10^{-2}\right.$ in a $100 \times 100$ lattice $)$; Fig. $\left.1(\mathrm{c})\right]$, rather different evolutionary outcomes are observed, for both the spatial model [Figs. 1(c)(i) and 1(c)(iii)] and the aspatial model [Figs. 1(c)(ii) and 1(c)(iv)]. In this situation, the long-term strategy frequencies are very similar whether interactions are local [Fig. 1(c)(iii)] or well-mixed [Fig. 1(c)(iv)]. Additionally, the high mutation results in a smooth transition in strategy frequencies between low and high $r$ [Figs. 1(c)(iii) and 1(c)(iv)], in contrast to low or absent mutation where much more abrupt changes in mean strategy frequency are noted [Figs. 1(a)(iii), 1(a)(iv), 1(b)(iii) and 1(b)(iv)]. In terms of tag frequencies in the spatial, tag-based Snowdrift game, high mutation prevents tag exclusion, and, indeed, promotes tag equality, at levels of $r$ that often resulted in the exclusion of one or the other tag in situations with low or no mutation [Fig. 1(c)(i)]. The same cannot be said of the aspatial version of the model, however, as one tag typically dominates the other, even when mutation is frequent, except at extremely low or extremely high values of $r$ [Fig. 1(c)(ii)]. Of course, this dominance 
is not complete as the "excluded" tag actually persists at a very low level due to its continual reintroduction via mutation.

\subsection{Aspatial and spatial five-tag models}

In the aspatial five-tag model with no mutation, all but one of the tags is excluded by a single victorious tag [Fig. 20(a)(ii)]. In this situation the remaining tags serve no purpose, and the tagbased Snowdrift game collapses to the non-tagbased Snowdrift game, with the combined frequency of $C$ - and $I$-strategists equal to $1-r$ [black line in Fig. 20(a)(iv)]. This is essentially the same outcome as in the two-tag Snowdrift game [Figs. 1(a)(ii) and 1(a)(iv)].

By contrast, in spatially structured populations, the results of the five-tag model with no mutation are very different from those of the twotag model with no mutation [compare Figs. 1(a)(i) and 1(a)(iii) with Figs. 20(a)(i) and 20(a)(iii)]. Specifically, in the five-tag model, $I$-strategists dominate the population over the entire range of the cost-benefit ratio of mutual cooperation, $r$ [Fig. 20(a)(iii)].

When mutation is present, aspatial and spatial five-tag models generally behave very similarly to two-tag models, albeit with a greater frequency of $I$-strategists [compare Figs. 1(b) and 1(c) with Figs. 20(b) and 20(c)]. Overall, these results suggest the hypothesis that tag diversity promotes nepotistic intra-tag conditional cooperation in tag-based systems.

\section{Discussion}

\subsection{Spatial interactions and their effects on tags and strategies}

As in non-tag-based systems (e.g. [Nowak \& May, 1992, 1993; Nowak et al., 1994a; Hauert, 2002; Hauert \& Doebeli, 2004; Hauert, 2006; Doebeli \& Hauert, 2005; Langer et al., 2008]) and in tagbased systems based on the Prisoner's Dilemma (e.g. [Jansen \& van Baalen, 2006; Laird, 2011]), spatial population structure plays an important role in the tag and strategy dynamics of the Snowdrift game (Figs. 1, 2 and 20). In terms of tags, spatial structure promotes tag coexistence in most instances [e.g. compare Figs. 1(a)(i) and 1(a)(ii); also see Fig. 2(a)]. Indeed, the models predict that when payoffs are according to the Snowdrift game, tag-based mechanisms of cooperation are only likely to be found in structured populations. (While $I$-strategists persist in aspatial populations, the lack of tag diversity means that they typically act exactly as if they were unconditional cooperators.) Here, I examine spatial structure, but it is likely that social network structure (i.e. as in evolutionary graph theory [Lieberman et al., 2005; Szabó \& Fáth, 2007; Taylor et al., 2007]) would produce similar results relative to unstructured populations; spatial structure on a square lattice can be considered a special case of a regular network.

In terms of strategies, spatial structure allows $I$-strategists to comprise most of the population in two-tag systems for all except the highest possible values of $r$ [e.g. Figs. 1(a)(iii) and 2(b)], and certainly higher than in the aspatial model [e.g. Fig. 1(a)(iv)], at least when the mutation rate is not extremely high [e.g. Fig. 1(c)(iii)]. Additionally, at very low values of $r, I$-strategists can dominate almost completely in the absence of mutation [e.g. Figs. 1(a)(iii) and 2(b)]. Thus, spatially local interactions can promote conditional cooperation in the two-tag Snowdrift game. This effect is even more prominent in the spatial five-tag Snowdrift game, in which $I$-strategists comprise the majority of the population for almost all of the conditions examined (Fig. 20).

\subsection{Phase transitions in the two-tag spatial Snowdrift game}

As in other studies of evolutionary game theory (e.g. [Szolnoki et al., 2009; Szolnoki \& Perc, 2010; Helbing et al., 2010b; Szolnoki et al., 2011]), the spatial two-tag Snowdrift game admits phase transitions across which the dynamics and the population composition change qualitatively [e.g. Figs. 1(a)(i), 1(a)(iii), 2(a) and 2(b)]. Transitioning across $r_{1}$ i.e. between Regions I and II - populations go from being composed of predominantly $I$-strategists (with a small number of subsisting $C$-strategists) to being composed of $I$ and $D$. Interestingly, this transition is the same as the lesser of the two identified by Hauert and Doebeli [2004] in their analysis of the non-tag-based Snowdrift game (Figs. 1(a)(i), 1(a)(iii), 2(a) and 2(b); compare with Fig. 1(b) in [Hauert \& Doebeli, 2004]). Thus, in the vicinity of $r_{1}$, there is either only one tag remaining, or, if two remain, they are sufficiently spatially segregated so that $I$-strategists are effectively equivalent to 
unconditional cooperators $(C)$ at single-tag locales, and the game approximates the spatial non-tagbased Snowdrift game. This means that uncooperative $D$-strategists can abruptly invade at the same value of $r$ as in Hauert and Doebeli's formulation, causing the change in population composition associated with this transition.

At $r_{2}$ (transition between Regions II and III), the population remains composed of $I$ - and $D$ strategists; however, the abrupt onset of nontransitive spatial invasion above $r_{2}$ allows both tags to coexist in the long term due to negative frequency-dependence. Indeed, as $r$ decreases through Region III, the period of the cyclic dynamics that emerges from this nontransitivity appears to approach infinity asymptotically at $r_{2}$ [Fig. 15(a)], signaling the lower boundary where such cyclic dynamics can occur without having one tag's $I-D$ mixture exclude the other's. The result is a phase transition that is discontinuous in terms of both tag coexistence [Figs. 1(a)(i) and 2(a)] and the dynamical behavior of the strategies [Figs. 1(a)(iii) and $2(\mathrm{~b})]$.

Proceeding across $r_{3}$ (transition between Regions III and IV), E-strategists abruptly persist at low frequencies along with the cycling $I$ and $D$-strategists. However, this phenomenon only occurs over a small range of cost-benefit ratios, as $E$-strategists abruptly cease to persist above $r_{4}$ (transition between Regions IV and V). Thus, $r_{3}$ and $r_{4}$ represent, respectively, the lower and upper limits of cost-benefit ratios that allow $E$-strategists to outcompete same-tagged $I$-strategists while they are concurrently outcompeted by different-tagged $I$ - and $D$-strategists.

\subsection{Comparison with tag-based Prisoner's Dilemma}

The results can also be compared to results from a similar model set-up, but instead using the payoff structure defined by the Prisoner's Dilemma [Laird, 2011]. The most striking difference is that the spatial tag-based Snowdrift game typically does not result in the evolution of traitorousness (i.e. $E$ strategists) except at very low levels that emerge over a small range of $r$-values [e.g. Region IV; Figs. 1(a)(iii) and 2(b)], or when it is buoyed up by very high mutation [Figs. 1(c)(iii) and 20(c)(iii)]. This is contrary to the spatial two-tag Prisoner's Dilemma, in which traitorousness can come to complete dominance at relatively low values of $r$ and $\mu$
[Laird, 2011]. In the aspatial two-tag model, Estrategists did have mean frequencies as high as 0.62 [Fig. 1(a)(iv)]; however, this is a trivial result, because it occurs when one of the tags has gone extinct, rendering $E$-strategists' conditionally cooperative behavior irrelevant. It may be the case, therefore, that widespread traitorousness is symptomatic of interactions based on the Prisoner's Dilemma rather than the Snowdrift game. Additionally, in the spatial, two-tag Prisoner's Dilemma, there is a large range of $r$ values for which unconditional defection $(D)$ dominates [Laird, 2011]. This phenomenon is absent in the tag-based Snowdrift game. This is perhaps not surprising in light of the fact that in the Prisoner's Dilemma game, mutual defection is the evolutionarily stable strategy, such that cooperation can only be "rescued" by spatial population structure when the cost of cooperation is sufficiently low, whereas in the Snowdrift game, even well-mixed populations are expected to have a mixture of cooperators and defectors at equilibrium.

\section{Conclusions}

In conclusion, the spatial, tag-based Snowdrift game produces an array of qualitatively distinct dynamical patterns, the occurrence of which depends on the cost-benefit ratio of mutual cooperation, $r$. These patterns, which emphasize the success of the intratag cooperation strategy in both two- and multitag models, demonstrate that the green-beard effect has applications beyond the traditional Prisoner's Dilemma game. Future work should test the robustness of this conclusion in light of various model features that may influence population-level outcomes (e.g. different update rules, different population sizes, more tags, iterated interactions, mixed- or otherwise more sophisticated strategies, different lattice geometries or network topologies, evolving networks and coevolutionary rules, strong versus weak selection, etc.; see [Szabó \& Fáth, 2007; Roca et al., 2009b, 2009a; Perc \& Szolnoki, 2010] for details).

\section{Acknowledgments}

I thank two anonymous reviewers for their helpful comments. Funding for this research was provided by the Natural Sciences and Engineering Research Council (Canada) and by the University of Lethbridge. 


\section{References}

Antal, T., Ohtsuki, H., Wakeley, J., Taylor, P. D. \& Nowak, M. A. [2009] "Evolution of cooperation by phenotypic similarity," Proc. Natl. Acad. Sci. USA 106, 8597-8600.

Axelrod, R. \& Hamilton, W. D. [1981] "The evolution of cooperation," Science 211, 1390-1396.

Axelrod, R., Hammond, R. A. \& Grafen, A. [2004] "Altruism via kin-selection strategies that rely on arbitrary tags with which they coevolve," Evolution 58, 1833-1838.

Axelrod, R. [2006] The Evolution of Cooperation, Revised edition (Basic Books, Cambridge, USA).

Chen, X.-J. \& Wang, L. [2010] "Effects of cost threshold and noise in spatial Snowdrift games with fixed multiperson interactions," Europhys. Lett. 90, 38003.

Dawkins, R. [1976] The Selfish Gene (Oxford University Press, Oxford).

Dawkins, R. [1982] The Extended Phenotype (Oxford University Press, Oxford).

Doebeli, M. \& Hauert, C. [2005] "Models of cooperation based on the Prisoner's Dilemma and the Snowdrift game," Ecol. Lett. 8, 748-766.

Du, W.-B., Cao, X.-B., Hu, M.-B. \& Wang, W.-X. [2009] "Asymmetric cost in Snowdrift game on scale-free networks," Europhys. Lett. 87, 60004.

Durrett, R. \& Levin, S. A. [1994] "Stochastic spatial models: A user's guide to ecological applications," Phil. Trans. R. Soc. Lond. B 343, 329-350.

Gintis, H. [2009] Game Theory Evolving, Second edition (Princeton University Press, Princeton, NJ).

Hamilton, W. D. [1964] "The genetical evolution of social behaviour. I and II," J. Theor. Biol. 7, 1-52.

Hammond, R. A. \& Axelrod, R. [2006a] "Evolution of contingent altruism when cooperation is expensive," Theor. Popul. Biol. 69, 333-338.

Hammond, R. A. \& Axelrod, R. [2006b] "The evolution of ethnocentrism," J. Conflict Resol. 50, 926-936.

Hauert, C. [2002] "Effects of space in $2 \times 2$ games," Int. J. Bifurcation and Chaos 12, 1531-1548.

Hauert, C. \& Doebeli, M. [2004] "Spatial structure often inhibits the evolution of cooperation in the Snowdrift game," Nature 428, 643-646.

Hauert, C. [2006] "Spatial effects in social dilemmas," J. Theor. Biol. 240, 627-636.

Helbing, D., Szolnoki, A., Perc, M. \& Szabó, G. [2010a] "Defector-accelerated cooperativeness and punishment in public goods games with mutations," Phys. Rev. E 81, 057104.

Helbing, D., Szolnoki, A., Perc, M. \& Szabó, G. [2010b] "Evolutionary establishment of moral and double moral standards through spatial interactions," PLoS Comput. Biol. 6, e1000758.
Hofbauer, J. \& Sigmund, K. [1998] Evolutionary Games and Population Dynamics (Cambridge University Press, Cambridge).

Jansen, V. A. A. \& van Baalen, M. [2006] "Altruism through beard chromodynamics," Nature 440, 663666.

Keller, L. \& Ross, K. G. [1998] "Selfish genes: A green beard in the red fire ant," Nature 394, 573-575.

Laird, R. A. [2011] "Green-beard effect predicts the evolution of traitorousness in the two-tag Prisoner's Dilemma," J. Theor. Biol. 288, 84-91.

Langer, P., Nowak, M. A. \& Hauert, C. [2008] "Spatial invasion of cooperation," J. Theor. Biol. 250, 634641.

Lieberman, E., Hauert, C. \& Nowak, M. A. [2005] "Evolutionary dynamics on graphs," Nature 433, 312-316.

Lima, F. W. S., Hadzibeganovic, T. \& Stauffer, D. [2009] "Evolution of ethnocentrism on undirected and directed Barbási-Albert networks," Physica A 388, 4999-5004.

Masuda, N. \& Ohtsuki, H. [2007] "Tag-based indirect reciprocity by incomplete social information," Proc. R. Soc. B 274, 689-695.

Maynard Smith, J. [1982] Evolution and the Theory of Games (Cambridge University Press, Cambridge, $\mathrm{UK})$.

Maynard Smith, J. \& Szathmáry, E. [1997] The Major Transitions of Evolution (Oxford University Press, NY).

Maynard Smith, J. \& Szathmáry, E. [1999] The Origins of Life: From the Birth of Life to the Origins of Language (Oxford University Press, Oxford).

Nowak, M. A. \& May, R. M. [1992] "Evolutionary games and spatial chaos," Nature 359, 826-829.

Nowak, M. A. \& May, R. M. [1993] "The spatial dilemmas of evolution," Int. J. Bifurcation and Chaos 3, $35-78$.

Nowak, M. A., Bonhoeffer, S. \& May, R. M. [1994a] "More spatial games," Int. J. Bifurcation and Chaos 4, 33-56.

Nowak, M. A., Bonhoeffer, S. \& May, R. M. [1994b] "Spatial games and the maintenance of cooperation," Proc. Natl. Acad. Sci. USA 91, 4877-4881.

Nowak, M. A. \& Sigmund, K. [1998] "Evolution of indirect reciprocity by image scoring," Nature 393, 573577.

Nowak, M. A. \& Sigmund, K. [2004] "Evolutionary dynamics of biological games," Science 303, 793-799.

Nowak, M. A. [2006a] Evolutionary Dynamics: Exploring the Equations of Life (Harvard University Press, Cambridge).

Nowak, M. A. [2006b] "Five rules for the evolution of cooperation," Science 314, 1560-1563. 
Nowak, M. \& Highfield, R. [2011] SuperCooperators: Altruism, Evolution, and Why We Need Each Other to Succeed (Free Press, NY).

Perc, M. [2007] "Uncertainties facilitate aggressive behavior in a spatial hawk-dove game," Int. J. Bifurcation and Chaos 17, 4223-4227.

Perc, M. \& Szolnoki, A. [2010] "Coevolutionary games A mini review," Biosystems 99, 109-125.

Queller, D. D., Ponte, E., Bozzaro, S. \& Straussmann, J. E. [2003] "Single-gene green beard effects in the social amoeba Dictyostelium dicoideum," Science 299, 105-106.

Riolo, R. L., Cohen, M. D. \& Axelrod, R. [2001] "Evolution of cooperation without reciprocity," Nature $\mathbf{4 1 4}$, 441-443.

Riolo, R. L., Cohen, M. D. \& Axelrod, R. [2002] "Does similarity breed cooperation? Reply," Nature 418, 500.

Roberts, G. \& Sherratt, T. N. [2002] "Does similarity breed cooperation?" Nature 418, 499-500.

Roca, C. P., Cuesta, J. A. \& Sánchez, A. [2009a] "Effect of spatial structure on the evolution of cooperation," Phys. Rev. E 80, 043106.

Roca, C. P., Cuesta, J. A. \& Sánchez, A. [2009b] "Evolutionary game theory: Temporal and spatial effects beyond replicator dynamics," Phys. Life Rev. 6, 208249.

Sherratt, T. N., Roberts, G. \& Kassen, R. [2009] "Evolutionary stable investment in products that confer both an individual benefit and public good," Front. Biosci. E1, 340-347.

Sherratt, T. N. \& Wilkinson, D. M. [2009] Big Questions in Ecology and Evolution (Oxford University Press, Oxford).

Smukalla, S., Caldara, M., Pochet, N., Beauvais, A., Gaudagnini, S., Yan, C., Vinces, M. D., Jansen, A., Prevost, M. C., Latgé, J.-P., Fink, G. R., Foster, K. R. \& Verstrepen, K. J. [2008] "FLO1 is a variable green beard gene that drives biofilm-like cooperation in budding yeast," Cell 135, 726-737.

Souza, M. O., Pacheco, J. M. \& Santos, F. C. [2009] "Evolution of cooperation under $N$-person Snowdrift games," J. Theor. Biol. 260, 581-588.

Sugden, R. [1986] The Economics of Rights, Cooperation and Welfare (Blackwell, Oxford).

Szabó, G. \& Fáth, G. [2007] "Evolutionary games on graphs," Phys. Rep. 446, 97-216.

Szolnoki, A. \& Perc, M. [2008] "Coevolution of teaching activity promotes cooperation," New J. Phys. 10, 043036.

Szolnoki, A. \& Perc, M. [2009] "Resolving social dilemmas on evolving random networks," Europhys. Lett 86, 30007.

Szolnoki, A., Perc, M. \& Szabó, G. [2009] "Phase diagrams for three-strategy evolutionary Prisoner's Dilemma games on regular graphs," Phys. Rev. E 80, 056104.

Szolnoki, A. \& Perc, M. [2010] "Reward and cooperation in the spatial public goods game," Europhys. Lett. 92, 38003.

Szolnoki, A., Szabó, G. \& Perc, M. [2011] "Phase diagrams for the spatial public goods game with pool punishment," Phys. Rev. E 83, 036101.

Taylor, P. D., Day, T. \& Wild, G. [2007] "Evolution of cooperation in a finite homogeneous graph," Nature 447, 469-472.

Traulsen, A. \& Schuster, H. G. [2003] "Minimal model for tag-based cooperation," Phys. Rev. E 68, 046129.

Traulsen, A. \& Nowak, M. A. [2007] "Chromodynamics of cooperation in finite populations," PLoS ONE $\mathbf{3}$, $\mathrm{e} 270$.

Traulsen, A. [2008] "Mechanisms for similarity based cooperation," Eur. Phys. J. B 63, 363-371.

Wang, W.-X., Ren, J., Chen, G. \& Wang, B.-H. [2006] "Memory-based Snowdrift game on networks," Phys. Rev. E 74, 056113. 